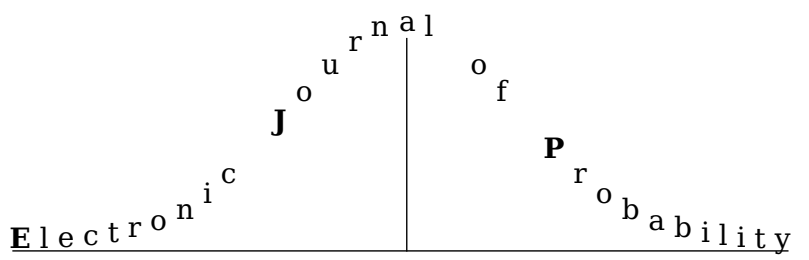

Electron. J. Probab. 26 (2021), article no. 132, 1-39.

ISSN: 1083-6489 https://doi.org/10.1214/21-EJP702

\title{
Lévy processes on smooth manifolds with a connection*
}

\author{
Aleksandar Mijatović ${ }^{\dagger} \quad$ Veno Mramor $^{\ddagger}$
}

\begin{abstract}
We define a Lévy process on a smooth manifold $M$ with a connection as a projection of a solution of a Marcus stochastic differential equation on a holonomy bundle of $M$, driven by a holonomy-invariant Lévy process on a Euclidean space. On a Riemannian manifold, our definition (with Levi-Civita connection) generalizes the Eells-ElworthyMalliavin construction of the Brownian motion and extends the class of isotropic Lévy process introduced in Applebaum and Estrade [3]. On a Lie group with a surjective exponential map, our definition (with left-invariant connection) coincides with the classical definition of a (left) Lévy process given in terms of its increments.

Our main theorem characterizes the class of Lévy processes via their generators on $M$, generalizing the fact that the Laplace-Beltrami operator generates Brownian motion on a Riemannian manifold. Its proof requires a path-wise construction of the stochastic horizontal lift and anti-development of a discontinuous semimartingale, leading to a generalization of Pontier and Estrade [32] to smooth manifolds with non-unique geodesics between distinct points.
\end{abstract}

Keywords: Lévy process on a smooth manifold; linear connection; holonomy bundle; Marcus stochastic differential equation; horizontal Lévy process; stochastic horizontal lift; stochastic anti-development.

MSC2020 subject classifications: 60G51; 58J65; 60J25.

Submitted to EJP on December 18, 2020, final version accepted on September 7, 2021.

Supersedes arXiv:2012.11633.

\section{Introduction}

Lévy processes on the Euclidean space $\mathbb{R}^{d}$ are fundamental and well-studied stochastic processes with càdlàg (right continuous with left limits) paths, characterized by the

\footnotetext{
${ }^{*} \mathrm{AM}$ and VM are supported by The Alan Turing Institute under the EPSRC grant EP/N510129/1; AM supported by EPSRC grant EP/P003818/1 and the Turing Fellowship funded by the Programme on Data-Centric Engineering of Lloyd's Register Foundation; VM supported by the PhD scholarship of Department of Statistics, University of Warwick.

${ }^{\dagger}$ Department of Statistics, University of Warwick, \& The Alan Turing Institute, UK.

E-mail: a.mijatovic@warwick.ac.uk

${ }^{\ddagger}$ Department of Statistics, University of Warwick, \& The Alan Turing Institute, UK.

E-mail: veno.mramor@warwick.ac.uk
} 
Lévy processes on smooth manifolds with a connection

independence and stationarity of their increments (see e.g. monographs $[7,37,26]$ and the references therein). Lévy processes have a natural generalization to Lie groups introduced in [19] and have been studied extensively over the last half a century (see monograph [28] for a modern account). The definition relies on the group structure of the state space, which allows to define a natural notion of the increment of a process. Since there is no natural notion of an increment on a Riemannian manifold $M$, [3] uses a geodesics-based approach to define isotropic Lévy processes on $M$. However, these two definitions are not entirely compatible: for example, if $M$ is a $d$-dimensional torus with a flat metric, isotropic Lévy processes form a strict subclass of Lévy processes on $M$, defined via its Lie group structure. The same phenomenon may be observed on the Euclidean space $\mathbb{R}^{d}$ or indeed any Lie group equipped with a left-invariant Riemannian metric.

The main contribution of the present paper is twofold. (I) By relaxing key restrictions from the definition of isotropic Lévy process on Riemannian manifolds from [3], we extend the definition of Lévy processes to smooth manifolds equipped with a connection. A fundamental property of Lévy processes is that they jump in the "same way" from any point in the state space. Our generalization is natural, since it is a connection (and not a metric) that is a required geometric structure for the comparison of jumps along geodesics at different points on the manifold. Moreover, a canonical connection is often available when there is no canonical metric. For example the class of Lévy processes induced by the left-invariant connection on a Lie group (with a surjective exponential map) coincides with the class of the classical (left) Lévy process. Furthermore, our definition with the Levi-Civita connection on a Riemannian manifold extends the class of isotropic Lévy processes, closing the aforementioned compatibility gap. (II) Our main theorem (Theorem 2.14 below) characterizes the law of a Lévy process on a smooth manifold with a connection via its infinitesimal generator, generalizing the classical result that the law of a Brownian motion on a Riemannian manifold is characterized by the Laplace-Beltrami operator.

Our definition generalizes the celebrated Eells-Elworthy-Malliavin construction of a Brownian motion on a $d$-dimensional Riemannian manifold, given as a projection of a horizontal Brownian motion in the orthonormal frame bundle. The horizontal Brownian motion is defined as a solution of the Stratonovich stochastic differential equation (SDE) on the orthonormal frame bundle, with the integrator a standard Brownian motion on $\mathbb{R}^{d}$ and the coefficients given by the fundamental horizontal vector fields. Consider a smooth manifold $M$ with a connection and a corresponding holonomy bundle (a subbundle of the frame bundle of $M$ ). We define a Lévy process $X$ on $M$ to be the projection of a horizontal Lévy process $U$, defined via an SDE on a holonomy bundle with the integrator a Lévy process $Y$ on $\mathbb{R}^{d}$ and the coefficients given by the fundamental horizontal vector fields in the tangent bundle of the holonomy bundle (see Section 2 below). Since $Y$ is typically discontinuous, we use the theory of Marcus SDEs [29, 25, 15, 4] in order to obtain the correct transformation properties under the change of coordinates on a smooth manifold, not possessed by the Stratonovich integral in the presence of jumps.

A Lévy process on $\mathbb{R}^{d}$ is a time-homogeneous Markov process. In order to ensure that the time-homogeneous Markov property holds for our process $X$ on $M$, the horizontal process $U$ on the holonomy bundle has to be (a) time-homogeneous Markov and (b) invariant under the natural action of the corresponding holonomy group. Since $U$ is a strong solution of a Marcus $\operatorname{SDE}$ with the integrator $Y$, requirement (a) forces the process $Y$ to be a Lévy process on $\mathbb{R}^{d}[20,14]$. Condition (b) is equivalent to the generating triplet of $Y$ being invariant under the action of a holonomy group on $\mathbb{R}^{d}$ (see (2.6) below).

Holonomy bundle and the corresponding holonomy group are not uniquely determined 
Lévy processes on smooth manifolds with a connection

by the connection on a connected smooth manifold $M$. However, all possible choices of holonomy bundles are diffeomorphic and the corresponding holonomy groups are conjugate Lie subgroups of the group of invertible matrices in $\mathbb{R}^{d} \otimes \mathbb{R}^{d}$. Thus, the choice of the holonomy bundle and the corresponding holonomy group does not affect the class of all Lévy processes on $M$ described by our definition (see Proposition 2.9 below).

The characterization of the law of a Lévy process $X$ via its infinitesimal generator requires a construction of the stochastic horizontal lift $U$ in the holonomy bundle and the stochastic anti-development $Y$ in $\mathbb{R}^{d}$, such that $U$ solves the Marcus SDE driven by $Y$ and is mapped into $X$ by the bundle projection. The jumps of the solution $U$ of the Marcus SDE are given via the flow of the horizontal vector fields, making $X$ jump along the geodesics in $M$. The main technical difficulty in constructing a stochastic horizontal lift $U$ from the generator of $X$ is that a jump of $X$ may be induced by many different jumps of $U$. This issue disappears when a manifold $M$ possesses a unique geodesic between any two points, as is assumed in [32], since then the structure of the Marcus SDE implies that the jumps of $X$ and $U$ are in one-to-one correspondence. However, this geometric assumption is quite restrictive as it excludes for example all compact Riemannian manifolds e.g spheres of any dimension. More precisely, compact Riemannian manifolds are bounded as metric spaces and on the other hand geodesically complete by the Hopf-Rinow theorem, which would imply unboundedness if the unique geodesic assumption were in place. To circumvent this issue in a general setting, we first append compatible "jump data" to the process $X$. We can then prove that $X$ and the "jump data" together uniquely determine the horizontal lift $U$ and the anti-development $Y$, see Theorem 2.19 below. Moreover, it is possible to construct on an enlarged probability space suitable "jump data" so that the anti-development $Y$ of the process $X$ is precisely a Lévy process on $\mathbb{R}^{d}$ with the generating triplet induced by the generator of $X$.

On Riemannian manifolds it is natural to consider the Levi-Civita connection which is uniquely determined by the metric. A holonomy group of this connection is a Lie subgroup of the orthogonal group implying that our new definition of Lévy processes is a genuine generalization of isotropic Lévy processes defined in [3] (see Section 3.1 below for details). In particular, since the holonomy group of the flat connection on $\mathbb{R}^{d}$ is trivial, our definition coincides with the classical definition of Lévy processes on $\mathbb{R}^{d}$. Lie groups do not necessarily possess a natural Riemannian metric, but have a natural choice of a left-invariant connection. In Section 3.2 below we show that this choice of a connection implies that a Lévy process satisfying our definition is a classical (left) Lévy process on a Lie group. If the exponential map of the Lie group is not surjective, then jumps which are not in the image of the exponential map cannot be expressed via the geodesics of the left-invariant connection, so (left) Lévy process having such jumps cannot be expressed via our definition. However, as soon as all jumps can be expressed in such a way, the (left) Lévy process can be expressed via our new definition (see Proposition 3.2). In particular, on the Lie group with surjective exponential map the two notions of Lévy processes coincide.

The remainder of the paper is structured as follows. In Section 2 we apply the theory of Marcus SDEs on manifolds to construct a Lévy process on a smooth manifold with a connection and state our main results. In Section 3 we discuss examples of Lévy processes on manifolds with a connection, including special cases of Riemannian manifolds and Lie groups as well as new examples with exceptional holonomy groups. The proofs of our main results are given in Section 4. Some facts from differential geometry used in our analysis are collected in Appendix A. 


\section{Construction and characterization of Lévy processes on mani- folds with a connection}

Let $M$ be a connected smooth $d$-dimensional manifold without boundary. Throughout we use the following notation: $C^{\infty}(M)$ denotes the space of smooth real valued functions on $M, T M$ is the tangent bundle on $M, \Gamma(T M)$ denotes the vector fields on $M$ (i.e. smooth sections of the tangent bundle), see [23] as a general reference for differential geometry. A càdlàg $M$-valued stochastic process $X=\left(X_{t}\right)_{t \in[0, \tau)}$ is a semimartingale on a stochastic interval $[0, \tau)$, where $\tau$ is a predictable stopping time if $f\left(X^{\tau_{n}}\right)$ is a real-valued semimartingale for any $f \in C^{\infty}(M)$ and $n \in \mathbb{N}$, where $X^{\tau_{n}}=\left(X_{t}^{\tau_{n}}\right)_{t \in \mathbb{R}_{+}}$with $X_{t}^{\tau_{n}}=X_{\min \left\{\tau_{n}, t\right\}}$ for $t \in \mathbb{R}_{+}$denotes a stopped process and stopping times $\left\{\tau_{n} ; n \in \mathbb{N}\right\}$ form an announcing sequence of $\tau$, i.e. $\tau_{n} \uparrow \tau$ as $n \rightarrow \infty$ and $\tau_{n}<\tau$ a.s. for every $n \in \mathbb{N}$. See $[33,21]$ as a general reference for standard (real) semimartingales and predictable stopping times. One easily checks using Itô's formula that if $M=\mathbb{R}^{d}$, this definition (with $\tau \equiv \infty$ ) is equivalent to the standard definition of a semimartingale.

The corresponding generalisation of Lévy processes is not as straightforward since on a general smooth manifold there is no suitable notion of an increment. An alternative approach, also used in [3], exploits the fact that points of a smooth manifold possess tangent spaces, which are real vector spaces providing a local linearization of the manifold. Each tangent space is isomorphic to $\mathbb{R}^{d}$, making it feasible to "develop" a Lévy process on a tangent space onto the manifold. Since at different points in $M$ the tangent spaces are not canonically isomorphic, for such a construction to work we need to be able to use curves in $M$ to "connect \& compare" different tangent spaces. This highlights the need for a (linear) connection on $M$. Our main aim is to show that this geometric structure is sufficient for a Lévy process on $M$ to be defined, circumventing the need for a Riemannian metric used in [3]. There is an intermediate step - we need to consider a horizontal process on the holonomy bundle, which is a solution of a specific Marcus SDE.

In Section 2.1 we recall basic properties of Marcus SDEs on manifolds. In Section 2.2 we apply Marcus SDEs to define a horizontal Lévy process on the holonomy bundle, which is then projected to a Lévy process on a manifold with a connection. Section 2.3 describes our class of Lévy processes via generators on $M$.

\subsection{Marcus SDE}

The Stratonovich integral which is used for SDEs on smooth manifolds with continuous semimartingale integrators does not obey the classical chain rule formula when the integrator has jumps. The first theory of SDEs with jumps on manifolds was introduced in [35] and allowed integration against Brownian motion and Poisson random measures with quite general jump coefficients. A more modern theory with the same class of integrators is studied in [24] and also deals with stochastic flows of diffeomorphisms. Technically, such an approach with a suitable choice of a jump coefficient could be used for our Definition 2.8 of Lévy processes on manifold where the integrator is a Euclidean Lévy process. However, we also wish to consider more general càdlàg semimartingale integrators, especially in the context of stochastic horizontal lifts and stochastic anti-developments (see Definition 2.15 and Theorem 2.19).

Hence, we will consider a unified approach and utilise a more general notion of a Marcus (or canonical) SDE which was introduced in the Euclidean spaces in [29] and was later generalized (still in Euclidean spaces) in [25] and allows integration against arbitrary càdlàg semimartingales. Due to correct change-of-variable properties Marcus SDE arises naturally in the context of smooth manifolds [15, 4, 3]. In order to describe the generalisation of Marcus SDE to smooth manifolds required in this paper, we first 
recall some notions from differential geometry.

A smooth curve $\gamma: I \subseteq \mathbb{R} \rightarrow M$ is an integral curve of a vector field $\mathcal{X} \in \Gamma(T M)$ if $\dot{\gamma}_{s}:=d \gamma_{s}\left(\left.\frac{d}{d t}\right|_{s}\right)=\mathcal{X}_{\gamma_{s}}$ holds for every $s \in I$, i.e. the velocity vector of the curve is equal to the value of the vector field $\mathcal{X}$ at the same point. As a consequence of the local existence and uniqueness theorem for systems of ordinary differential equations (ODEs), there exists a unique integral curve of $\mathcal{X}$ on the maximal time interval once the initial point is fixed. We say that a vector field is complete if all its integral curves are defined for all times in $\mathbb{R}$. This allows us to consider the flow of a vector field and a flow exponential map. A flow of a complete vector field $\mathcal{X}$ is a one-parameter group of diffeomorphisms $\Phi_{s}^{\mathcal{X}}: M \rightarrow M$ for any $s \in \mathbb{R}$, such that for any $p \in M$ the curve $s \mapsto \gamma_{\mathcal{X}}^{p}(s):=\Phi_{s}^{\mathcal{X}}(p)$ is a unique integral curve of $\mathcal{X}$ with $\gamma_{\mathcal{X}}^{p}(0)=p$. It is easy to see that we have the flow property $\Phi_{s}^{\mathcal{X}} \circ \Phi_{t}^{\mathcal{X}}=\Phi_{s+t}^{\mathcal{X}}$ for any $s, t \in \mathbb{R}$. We can also define the flow exponential map $\exp (\mathcal{X}):=\Phi_{1}^{\mathcal{X}}: M \rightarrow M$ which represents a diffeomorphism which moves points a unit time along the integral curves of a vector field $\mathcal{X}$.

Definition 2.1. Let $M$ be a smooth manifold, let $V_{1}, \ldots, V_{\ell} \in \Gamma(T M)$ be smooth vector fields on $M$ and let $W$ be an $\mathbb{R}^{\ell}$-valued càdlàg semimartingale. We call an $M$-valued semimartingale $X$ defined on a stochastic interval $[0, \tau)$ a solution of a Marcus SDE

$$
\mathrm{d} X_{t}=V_{i}\left(X_{t-}\right) \diamond \mathrm{d} W_{t}^{i}
$$

if for any $f \in C^{\infty}(M)$ it satisfies the equation

$$
\begin{aligned}
f\left(X_{t}\right)-f\left(X_{0}\right) & =\int_{0}^{t} V_{i} f\left(X_{s-}\right) \circ \mathrm{d} W_{s}^{i} \\
& +\sum_{0<s \leq t}\left(f\left(\exp \left(V_{i} \Delta W_{s}^{i}\right)\left(X_{s-}\right)\right)-f\left(X_{s-}\right)-V_{i} f\left(X_{s-}\right) \Delta W_{s}^{i}\right)
\end{aligned}
$$

for $0 \leq t<\tau$, where Itô's circle $\circ$ denotes the Stratonovich integral, $\Delta W_{s}=W_{s}-W_{s-}$ and exp is a flow exponential map.

Remark 2.2. (i) In (2.1) and (2.2), as well as in the rest of the paper, we always use Einstein's convention of summing over repeated indices, when they appear once as an upper and once as a lower index.

(ii) A more precise meaning of (2.2) is as follows. Consider an announcing sequence $\left\{\tau_{n} ; n \in \mathbb{N}\right\}$ of $\tau$. We require that for every $n \in \mathbb{N}$ the stopped process $X^{\tau_{n}}$ is a semimartingale and that equation (2.2) with $X$ exchanged by $X^{\tau_{n}}$ holds for every $t \in \mathbb{R}_{+}$.

(iii) The sum in the defining property (2.2) of a Marcus SDE can be interpreted as follows: the process $X$ jumps only if the integrator $W$ jumps and then the jump of $X$ occurs along an integral curve of a certain vector field, constructed as a linear combination of the driving vector fields with the coefficients given by the components of the jump of the integrator.

Proposition 2.3. Suppose that for every $a=a^{i} \mathrm{e}_{i} \in \mathbb{R}^{d}$ the vector field $a^{i} V_{i}$ is complete. Then there exists a unique solution $X$ of Marcus SDE (2.1) and it is a semimartingale defined on a maximal stochastic interval $[0, \tau)$, where $\tau$ is a predictable stopping time which is an explosion time ${ }^{1}$.

Remark 2.4. A solution process $X$ of Marcus SDE (2.1) exists even when vector fields are not complete. However, then the stopping time $\tau$ is not necessarily predictable and it might happen that $X$ "jumps" to the cemetery point $\partial$ and does not reach it continuously. To avoid these issues we will insist on the completeness assumption.

\footnotetext{
${ }^{1}$ If we consider a one-point compactification $\widehat{M}:=M \cup\{\partial\}$ this means that on the event $\{\tau<\infty\}$ as $t \rightarrow \tau$ we have $X_{t} \rightarrow \partial$ or equivalently $X$ exits all compacts in $M$.
} 
Since all vector fields on compact manifolds are complete, we immediately obtain the following corollary.

Corollary 2.5. If $M$ is compact, then the solution $X$ of Marcus SDE (2.1) is a semimartingale defined on the whole interval $[0, \infty)$.

A more general type of SDEs on manifolds, which includes (2.1), was considered in $[10,11]$. But for the sake of completeness and due to its simplicity, we give a direct proof of Proposition 2.3 in Section 4.1 below.

\subsection{Horizontal Lévy processes and Lévy processes on a smooth manifold with a connection}

Let $F(M)$ denote a frame bundle over $M$ (defined as a bundle of linear frames in [23, p. 56]) with a bundle projection $\pi: F(M) \rightarrow M$. The bundle $F(M)$ is a smooth manifold of dimension $d+d^{2}$ and the projection $\pi$ is a smooth map. A frame $u \in F(M)$, such that $\pi(u)=p$, is an ordered basis $u_{1}, \ldots, u_{d}$ of the tangent space $T_{p} M$. We may regard it as a linear isomorphism $u: \mathbb{R}^{d} \rightarrow T_{p} M$ given by

$$
u(x)=x^{i} u_{i} \text { for each } x=x^{i} e_{i} \in \mathbb{R}^{d},
$$

where $e_{1}, \ldots, e_{d}$ is a standard basis of $\mathbb{R}^{d}$. The general linear group $\mathrm{GL}(d)$, consisting of invertible matrices in $\mathbb{R}^{d} \otimes \mathbb{R}^{d}$, acts on $F(M)$ on the right by $R_{g}(u)=u g:=u \circ g$, where $g \in \mathrm{GL}(d)$ is considered as an automorphism of $\mathbb{R}^{d}$. This turns $F(M)$ into a principal fibre bundle (over $M$ ) with a structure group GL(d) (see definition in [23, §5, Ch. I]).

We assume that $M$ is equipped with a (linear) connection. A connection is given by a covariant derivative which prescribes how vector fields can be derived along other vector fields. A covariant derivative induces a direct sum splitting $T_{u} F(M)=H_{u} F(M) \oplus V_{u} F(M)$ of the tangent spaces of the frame bundle $F(M)$ at each frame $u$. Whereas vertical spaces $V_{u} F(M):=\operatorname{ker} d \pi_{u}$ are canonically defined, the horizontal spaces $H_{u} F(M)$ are induced by the covariant derivative and they are invariant by the differential of a right group action of GL $(d)$. This (invariant) splitting is what is classically defined as a (linear) connection in the frame bundle $F(M)$ in [23, Chs. II and III]. It is well-known that such an invariant splitting on $F(M)$ is equivalent to a covariant derivative, see [23, Thm. 7.5 in Ch. III]. More details about covariant derivatives and the induced splitting are given in Appendix A.

The horizontal vector spaces are of dimension $d$. Since $d \pi_{u}$ is surjective, the restricted map $d \pi_{u}: H_{u} F(M) \rightarrow T_{\pi(u)} M$ is an isomorphism, inducing the horizontal lift in $T_{u} F(M)$ of any tangent vector in $T_{\pi(u)} M$. Thus, for each $x \in \mathbb{R}^{d}$ there exists a canonical fundamental horizontal vector field $H_{x}$ on $F(M)$ (defined as a standard horizontal vector field in [23, p. 119]), uniquely determined by:

(H1) $H_{x}(u) \in H_{u} F(M)$,

(H2) $d \pi_{u}\left(H_{x}(u)\right)=u(x)$

for each $u \in F(M)$. It follows that $x \mapsto H_{x}$ is a linear map and we denote $H_{i}:=H_{e_{i}}$ for $e_{1}, \ldots, e_{d}$ the standard basis of $\mathbb{R}^{d}$. In order to be able to use Proposition 2.3 and avoid the non-predictability issues outlined Remark 2.4, we will assume that all the vector fields $H_{e}, e \in \mathbb{R}^{d}$ are complete, which is equivalent to geodesic completeness of the manifold $M$ (see paragraph preceding equation (A.3) for relation between geodesics and integral curves of fundamental horizontal vector fields).

Definition 2.6. Let $M$ be equipped with a connection which turns it into a geodesically complete manifold. An $F(M)$-valued semimartingale $U$ defined on a stochastic interval $[0, \tau)$ is called a horizontal Lévy process if there exists an $\mathbb{R}^{d}$-valued Lévy process $Y$ (see 
Lévy processes on smooth manifolds with a connection

[37, Def. 1.6] for a definition of $\mathbb{R}^{d}$-valued Lévy processes) such that $U$ is a solution of a Marcus SDE

$$
\mathrm{d} U_{t}=H_{i}\left(U_{t-}\right) \diamond \mathrm{d} Y_{t}^{i}, \quad U_{0}=u \in F(M),
$$

on the maximal (stochastic) interval $[0, \tau)$.

Remark 2.7. (i) Horizontal Lévy processes were originally introduced in [1] on the orthonormal bundle of the Riemannian manifold.

(ii) We require geodesic completeness so that the flow exponential map is well defined on complete vector fields $H_{x}, x \in \mathbb{R}^{d}$. Proposition 2.3 then shows that a horizontal Lévy process $U$ is uniquely determined by the Lévy process $Y$ and is defined on the maximal stochastic interval $[0, \tau)$, where the assumption on geodesic completeness guarantees that $\tau$ is predictable.

In the next step, we use the bundle projection $\pi$ and consider the projected process $X:=\pi(U)$ which is a candidate for a Lévy process on $M$. Since the integrator $Y$ of Marcus SDE (2.4) is a Lévy process, the horizontal Lévy process $U$ is always a strong Markov process [25, Thm. 5.1], but $X$ in general need not be. More precisely, if $X_{0}=p$ we only know that $U_{0}$ is a frame in the fibre above $p$, i.e. $U_{0}=u$ such that $\pi(u)=p$. But $U_{0}$ might be a different frame $v$ in the same fibre. The future evolution of $U$, and hence of $X$, could be vastly different in these two cases, $U_{0}=u$ or $v$. However, since $u$ and $v$ are in the same fibre, there exists $g \in \mathrm{GL}(d)$ such that $u=v g$. With this in mind, consider the process $\widetilde{U}:=U g$, which by Lemma 4.3 below solves the Marcus SDE

$$
\mathrm{d} \widetilde{U}_{t}=\left(R_{g}\right)_{*} H_{i}\left(\widetilde{U}_{t-}\right) \diamond \mathrm{d} Y_{t}^{i},
$$

where push-forward vector fields $\left(R_{g}\right)_{*} H_{i}$ are given in (4.8) below. Moreover, by Lemma A.2 in the appendix, we have $\left(R_{g}\right)_{*} H_{x}=H_{g^{-1} x}$ for every $x \in \mathbb{R}^{d}$. Thus, linearity of $x \mapsto H_{x}$ and Lemma 4.1 imply that $\widetilde{U}$ is also a solution of Marcus SDE

$$
\mathrm{d} \widetilde{U}_{t}=H_{i}\left(\widetilde{U}_{t-}\right) \diamond \mathrm{d} \widetilde{Y}_{t}^{i}
$$

with $\widetilde{U}_{0}=u g=v$ where $\widetilde{Y}:=g^{-1} Y$. Consider a solution $V$ of Marcus SDE (2.4) with initial condition $V_{0}$, which is driven by $Y$. Therefore, if $Y$ and $\widetilde{Y}$ were to have the same law, then (weak) uniqueness of solutions of Marcus SDEs shows that $\widetilde{U}$ and $V$ also have the same law, thus implying that the processes $\pi(U)=\pi(\widetilde{U})$ and $\pi(V)$ are equal in law. Hence by assuming the appropriate invariance of the integrator $Y$ we may use the result of Dynkin on transformations of Markov processes [13, Thm. 10.13] which ensures the Markovianity of the projected process $X$. Fortunately, as we will see below, we do not have to check such an invariance for every $g \in \mathrm{GL}(d)$, but only for elements of a specific subgroup - the holonomy group, which we now describe.

For any frame $u \in F(M)$, a connection on $M$ allows us to reduce the frame bundle $F(M)$ to a subbundle $P(u)$ known as the holonomy bundle. The holonomy bundle $P(u)$ is a principal fibre bundle over $M$ with a structure group given by the holonomy group $\operatorname{Hol}(u)$, a Lie subgroup of GL $(d)$ [23, Thm. 4.2 in Ch. II]. More precisely, a holonomy bundle $P(u)$ consists of all the frames in $F(M)$ connected to $u$ via horizontal curves in $F(M)$ (i.e. curves with velocity vectors in horizontal spaces). The essential property for our definition of Lévy processes is that the fundamental horizontal vector fields $H_{x}$, $x \in \mathbb{R}^{d}$, are tangent to the subbundle $P(u)$ (this follows by the definition of the holonomy bundle via horizontal curves), hence they can be interpreted as vector fields on $P(u)$. By Proposition 2.3, any horizontal Lévy process $U$ (a solution of Marcus SDE (2.4)) with $U_{0} \in P(u)$ stays in $P(u)$ for all times for which it is defined.Hence to use [13, Thm 10.13] which ensures that the projection $X=\pi(U)$ is Markov, it is sufficient to check that the integrator $Y$ is $\operatorname{Hol}(u)$-invariant, i.e. the processes $Y$ and $g Y$ are equal in law for every $g \in \operatorname{Hol}(u)$ or equivalently the characteristics of $Y$ satisfy (2.6) below. 
Lévy processes on smooth manifolds with a connection

Definition 2.8. Let $Y$ be a $\operatorname{Hol}(u)$-invariant Lévy process on $\mathbb{R}^{d}$ and let $U=\left(U_{t}\right)_{t \in[0, \tau)}$ be a horizontal Lévy process on the holonomy bundle $P(u)$ that solves Marcus SDE (2.4) with $U_{0} \in P(u)$ a.s. Then with $\pi$ the bundle projection as above, $X=\pi(U)$ is said to be a Lévy process on $M$.

Proposition 2.9. A process $X$ on $M$ satisfying Definition 2.8 is a Markov process and is a semimartingale on $[0, \tau)$. The class of all Lévy processes on a connected smooth manifold $M$ is invariant under the choice of the frame $u \in F(M)$ and the corresponding holonomy bundle $P(u)$.

Remark 2.10. (i) The holonomy bundle is a reduction of the frame bundle (see [23] for definition of a reduction). In fact, it is the smallest possible reduction compatible with the connection [36, Remark 1.7.14]. Hence we cannot in general reduce the holonomy group invariance of the Lévy integrator any further and the class of Lévy processes on the smooth manifold $M$ with a connection from Definition 2.8 is the largest possible.

(ii) Due to a particular form of Marcus SDE (2.4), we know that $U_{t}=\exp \left(H_{i} \Delta Y_{t}^{i}\right)\left(U_{t-}\right)$ holds for any $t<\tau$, where exp is a flow exponential map (see Section 2.1). Then equation (A.3) shows that $X_{t}=\operatorname{Exp}_{X_{t-}}\left(U_{t-} \Delta Y_{t}\right)$ holds for any $t<\tau$, where $\operatorname{Exp}_{p}$ denotes a geodesic exponential map based at a point $p \in M$ (see Appendix A). Therefore, jumps of $X$ occur only along geodesics.

(iii) In the special case of Riemannian manifolds, [30, Sec. 3] considered a holonomy bundle reduction and obtained similar results to Proposition 2.9. More details about the Riemannian case are given in Section 3.1.

(iv) Note that if a Lévy process $Y$ is adapted to filtration $\left(\mathcal{F}_{t}\right)_{t \in \mathbb{R}_{+}}$, then also the horizontal Lévy process $U$ and the Lévy process $X$ on $M$ are adapted to the same filtration. However, even when we consider the natural filtration $\left(\mathcal{F}_{t}^{Y}\right)_{t \in \mathbb{R}_{+}}$of $Y$, it could happen that the natural filtrations of $U$ and $X$ are strictly smaller than $\left(\mathcal{F}_{t}^{Y}\right)_{t \in \mathbb{R}_{+}}$.

\subsection{Generators of Lévy processes on manifolds with a connection}

In this section we give a characterisation via generators of Lévy processes on smooth manifolds. The first step is to analyse the generators of horizontal Lévy processes.

Lemma 2.11. The (extended) generator of a horizontal Lévy process $U$, which solves Marcus SDE (2.4), is given by

$$
\begin{aligned}
\mathscr{L}_{U}(f)(v) & =H_{b} f(v)+\frac{1}{2} a^{i j} H_{i} H_{j} f(v) \\
& +\int_{\mathbb{R}^{d} \backslash\{0\}}\left(f\left(\exp \left(H_{x}\right)(v)\right)-f(v)-\mathbb{1}(|x|<1) x^{i} H_{i} f(v)\right) \nu(\mathrm{d} x)
\end{aligned}
$$

for any frame $v \in P(u)$, where $(a, \nu, b)$ is a generating triplet [37, p. 65] of a Lévy integrator $Y$ associated with a cut-off function $\chi(x)=\mathbb{1}(|x|<1)$ and the domain of $\mathscr{L}_{U}$ includes smooth functions on the holonomy bundle $P(u)$.

Recall that a Lévy process $Y$ is $\operatorname{Hol}(u)$-invariant if the laws of processes $Y$ and $g Y$ are the same for every $g \in \operatorname{Hol}(u)$. This holds if and only if the generating triplet $(a, \nu, b)$ of $Y$ 
satisfies

$$
\begin{aligned}
& \left.\nu \text { is } g \text {-invariant (i.e. } g_{*} \nu=\nu\right) \\
& g a g^{\top}=a \\
& g b=b+\int_{\mathbb{R}^{d} \backslash\{0\}}\left(\mathbb{1}\left(\left|g^{-1} x\right|<1\right)-\mathbb{1}(|x|<1)\right) \nu(\mathrm{d} x)
\end{aligned}
$$

for every $g \in \operatorname{Hol}(u)$, where the push-forward measure $g_{*} \nu$ is defined by $g_{*} \nu(A):=$ $\nu\left(g^{-1}(A)\right)$ for every Borel measurable set $A \in \mathfrak{B}\left(\mathbb{R}^{d}\right), a$ and $g$ are interpreted as a matrices in $\mathbb{R}^{d} \otimes \mathbb{R}^{d}$ and ${ }^{\top}$ denotes matrix transpose. Note that when $\operatorname{Hol}(u)$ is a subgroup of the orthogonal group $\mathrm{O}(d)$, the condition on the drift reduces to $g b=b$.

Since Lévy process $X$ is given as a projection of a horizontal Lévy process $U$, its generator is a "push-forward" of a generator (2.5) and invariance conditions (2.6) ensure it is indeed a well-defined operator.

Proposition 2.12. The generator $\mathscr{L}_{X}$ of a Lévy process $X$ on a smooth manifold $M$ with a connection is given by

$$
\begin{aligned}
\mathscr{L}_{X}(f)(p) & =H_{b}(f \circ \pi)(v)+\frac{1}{2} a^{i j} H_{i} H_{j}(f \circ \pi)(v) \\
& +\int_{\mathbb{R}^{d} \backslash\{0\}}\left(f\left(\operatorname{Exp}_{p}(v x)\right)-f(p)-\mathbb{1}(|x|<1) x^{i} H_{i}(f \circ \pi)(v)\right) \nu(\mathrm{d} x),
\end{aligned}
$$

where $v$ is any frame (in the holonomy bundle $P(u)$ ) with $\pi(v)=p$ and $\operatorname{Exp}_{p}$ is a geodesic exponential map based at $p$. In particular, (2.7) does not depend on the choice of $v$. The domain of the generator $\mathscr{L}_{X}$ comprises functions $f$ on $M$ such that $f \circ \pi$ is in the domain of the generator $\mathscr{L}_{U}$.

Remark 2.13. In special cases the formula in (2.7) can be simplified.

(i) Suppose that $-I_{d} \in \operatorname{Hol}(u)$, where $I_{d}$ is the identity matrix in $\mathbb{R}^{d} \otimes \mathbb{R}^{d}$. Then $-v \in P(u)$, and hence $H_{i}(f \circ \pi)(-v)=-H_{i}(f \circ \pi)(v)$, and (2.6) implies $b=0$. Assume further that $a=0$. Then by (2.7) applied with $v$ and $-v$ yields

$$
\begin{aligned}
\mathscr{L}_{X}(f)(p) & =\frac{1}{2} \int_{\mathbb{R}^{d} \backslash\{0\}}\left(f\left(\operatorname{Exp}_{p}(v x)\right)-f(p)-\mathbb{1}(|x|<1) x^{i} H_{i}(f \circ \pi)(v)\right) \nu(\mathrm{d} x) \\
& +\frac{1}{2} \int_{\mathbb{R}^{d} \backslash\{0\}}\left(f\left(\operatorname{Exp}_{p}(-v x)\right)-f(p)+\mathbb{1}(|x|<1) x^{i} H_{i}(f \circ \pi)(v)\right) \nu(\mathrm{d} x) \\
& =\frac{1}{2} \int_{T_{p} M}\left(f\left(\operatorname{Exp}_{p}(y)\right)-2 f(p)+f\left(\operatorname{Exp}_{p}(-y)\right)\right) \nu_{p}(\mathrm{~d} y),
\end{aligned}
$$

where the push-forward measure $\nu_{p}:=v_{*} \nu$ is independent of $v$ by (2.6), implying the expression for the jump part of the generator obtained in [6]. There are many examples of Riemannian manifolds with a Levi-Civita connection where $-I_{d} \in \operatorname{Hol}(u)$, e.g. when $d$ is even and $\operatorname{Hol}(u)=\mathrm{SO}(d)$ is the special orthogonal group. There also exist manifolds with a connection not induced by the Riemannian metric such that $-I_{d} \in \operatorname{Hol}(u)$, see Section 3.3.1.

(ii) In the case the stochastic anti-development Lévy process $Y$ is $\mathrm{O}(d)$-invariant in the sense of (2.6), the generating triplet equals $\left(\alpha I_{d}, \nu, 0\right)$ for some $\alpha \geq 0$ and an $\mathrm{O}(d)$ invariant Lévy measure $\nu$. If $M$ is a Riemannian manifold equipped with the LeviCivita connection, the holonomy bundle is a subbundle of the orthonormal frame bundle and the horizontal Laplace operator satisfies $\sum_{i=1}^{d} H_{i}^{2}(f \circ \pi)(v)=\Delta_{M} f(p)$ 
for any orthonormal frame $v$ in the fibre above $p$, where $\Delta_{M}$ is the Laplace-Beltrami operator on $M$. Thus (2.7) and (2.8) imply

$$
\mathscr{L}_{X}(f)(p)=\frac{\alpha}{2} \Delta_{M} f(p)+\frac{1}{2} \int_{T_{p} M}\left(f\left(\operatorname{Exp}_{p}(y)\right)-2 f(p)+f\left(\operatorname{Exp}_{p}(-y)\right)\right) \nu_{p}(\mathrm{~d} y),
$$

which equals the generator of an isotropic Lévy process in [3], where the improper integrals in the jump part were used implicitly in [3, Eq. (3.6)].

For the formula (2.7) to be of use, it is crucial to prove that any Markov process $X$ on $M$ with the generator $\mathscr{L}_{X}$ is indeed a Lévy process on $M$ satisfying Definition 2.8. Put differently, we need to construct an $\mathbb{R}^{d}$-valued Lévy process $Y$ such that $X$ can be represented via the Marcus SDE in Definition 2.8. Typically, we will not be able to construct $Y$ on the same probability space on which $X$ is defined and we will need to consider an extended probability space. Additionally, the filtration $\left(\mathcal{H}_{t}\right)_{t \in \mathbb{R}_{+}}$, to which $X$ is adapted, will also have to be extended to a larger filtration $\left(\mathcal{G}_{t}\right)_{t \in \mathbb{R}_{+}}$and this will constitute a strong Markov extension (see [8, Def. 2.47]). Existence of the appropriate process $Y$ on the extended probability space is the content of the following theorem.

Theorem 2.14. Let $X$ be a Markov process on $M$ adapted to $\left(\mathcal{H}_{t}\right)_{t \in \mathbb{R}_{+}}$with a generator $\mathscr{L}_{X}$ given by (2.7). Then there exists an extended probability space and on it an $\mathbb{R}^{d}$ valued Lévy process $Y$ adapted to a an extended filtration $\left(\mathcal{G}_{t}\right)_{t \in \mathbb{R}_{+}}$which forms a strong Markov extension, such that $X=\pi(U)$ where $U$ is a horizontal Lévy process solving Marcus SDE (2.4).

\subsection{Construction of horizontal lifts and anti-developments for discontinuous processes}

As mentioned above, the proof of Theorem 2.14 requires us to construct an integrator of Marcus SDE (2.4), such that the solution of this Marcus SDE is projected into a given process. In order to highlight the difficulties arising due to the presence of jumps, it is natural to consider such a construction in a more general setting of horizontal lifts and stochastic anti-developments of general semimartingales. Theorem 2.19 below, which resolves these problems and we hope is of independent interest, is a key step in the proof of Theorem 2.14.

Definition 2.15. An $F(M)$-valued semimartingale $U$ on $[0, \tau)$ is said to be horizontal if there exists an $\mathbb{R}^{d}$-valued semimartingale $W=\left(W_{t}\right)_{t \in \mathbb{R}_{+}}$with $W_{0}=0$, such that $U$ is a solution of a Marcus SDE

$$
\mathrm{d} U_{t}=H_{i}\left(U_{t-}\right) \diamond \mathrm{d} W_{t}^{i}
$$

on the stochastic interval $[0, \tau)$. The semimartingale $W$ is then called a stochastic antidevelopment of $U$ (or of its projection $X=\pi(U)$ ). Additionally, if $X$ is an $M$-valued semimartingale on $[0, \tau)$, then a horizontal semimartingale $U$ is called a stochastic horizontal lift of $X$ if $\pi(U)=X$.

Remark 2.16. (i) If $W$ in Definition 2.15 is in addition a Lévy process on $\mathbb{R}^{d}$, we obtain Definition 2.6.

(ii) Only times up to $\tau$ are used in Marcus SDE (2.9) so it suffices for $W$ to be semimartingale defined only on $[0, \tau)$.

If $X$ is a continuous $M$-valued semimartingale on $[0, \tau)$, then the stochastic antidevelopment and the stochastic horizontal lift of $X$ always exist and are unique (on $[0, \tau)$ ) once the initial frame $U_{0}$ satisfying $\pi\left(U_{0}\right)=X_{0}$ has been chosen [18, Sec. 2.3]. However, in the discontinuous case, the uniqueness of the stochastic anti-development and the existence of the stochastic horizontal lift may fail. To illustrate some potential 
Lévy processes on smooth manifolds with a connection

issues posed by jumps, we first focus on the stochastic anti-development of a horizontal semimartingale $U$, which exists by definition. Uniqueness, however, is related to whether at a jump time $s$ of $U$ there exists a unique fundamental horizontal vector field (and hence a unique jump of the integrator corresponding to it), whose flow exponential map connects the two endpoints $U_{s-}$ and $U_{s}$ of the jump. A simple example where uniqueness fails is when $M=\mathbb{S}^{1}:=\left\{z \in \mathbb{R}^{2} ;|z|=1\right\}$ is a unit circle equipped with the Levi-Civita connection induced by the standard Riemannian metric of the ambient space $\mathbb{R}^{2}$. Then one easily sees that $F\left(\mathbb{S}^{1}\right)$ is actually diffeomorphic to $\mathbb{S}^{1} \times(\mathbb{R} \backslash\{0\})$ and the fundamental horizontal vector field is $H_{1}=\left(\frac{d}{d \theta}, 0\right)$. Hence $\exp \left(2 \pi k H_{1}\right)(u)=u$ for $k \in \mathbb{Z}$, implying that no jump occurs in the case where the integrator has a jump of a size $2 \pi k$. We can then take an arbitrary real semimartingale $W$ and let $U$ be a horizontal semimartingale satisfying $\mathrm{d} U_{t}=H_{1}\left(U_{t-}\right) \diamond \mathrm{d} W_{t}$. But then $U$ is also a solution of a Marcus SDE $\mathrm{d} U_{t}=H_{1}\left(U_{t}\right) \diamond \mathrm{d} \widetilde{W}_{t}$, where $\widetilde{W}=W+2 \pi N$ and $N$ is a Poisson process. In particular, this shows that a continuous horizontal process may have a discontinuous stochastic anti-development (its continuous stochastic anti-development is unique).

On the other hand, the stochastic horizontal lift of a semimartingale $X$ need not exist. Jumps of a horizontal semimartingale occur only along integral curves of fundamental horizontal vector fields, hence jumps of its projection can only occur along geodesics i.e. the end-point of a jump has to be given as a point in the image of a geodesic exponential map based at the start point of the jump. But it could be the case that between the two endpoints $X_{t-}$ and $X_{t}$ of the jump there does not exist a geodesic. For example, we can take as our manifold a punctured plane $\mathbb{R}^{2} \backslash\{0\}$, which is an open subspace of the Euclidean plane, and since there are no geodesics (straight lines) between $x$ and $-x$, a process making such jumps cannot have a stochastic horizontal lift.

Furthermore, even when suitable geodesics exist, they might not be unique, implying the non-uniqueness of horizontal lifts. A typical example is given by the two antipodal points on a sphere and there exists a whole one parameter family of possible geodesics (along great circles). Even between two arbitrary points on a sphere one could travel several times around the sphere along a great circle before stopping at the endpoint, thus breaking uniqueness. Another similar issue related to non-uniqueness of geodesics is that even when we observe no jump of a semimartingale, we could still sometimes force its horizontal lift to jump in a way that would not manifest itself as a jump after projection.

Under suitable assumptions, these issues can be dispensed with. In the setting of Riemannian manifolds, [32] considered semimartingales such that there exists a unique geodesic between the two end points of any jump. They were able to show that for such processes the stochastic horizontal lift exists and is unique once the initial frame is fixed, and the same is true for the stochastic anti-development. As mentioned in the introduction, the assumption of unique geodesics is quite strict, excluding compact Riemannian manifolds, such as spheres and projective spaces. In order to deal with general smooth manifolds we provide an alternative approach based on Theorem 2.19 below.

The main issues when constructing horizontal lift and anti-development of a semimartingale $X$ concern the jumps of $X$. It is not sufficient to simply know that a jump occurred at some time $s$, but we also need to know how it happened, i.e. which specific geodesic was used to induce the jump. Geodesics can be parametrized via a geodesic exponential map, hence we need to specify a tangent vector $v \in T_{X_{s-}} M$ such that $X_{s}=\operatorname{Exp}_{X_{s-}}(v)$ holds. Typically, we cannot uniquely determine such a vector from $X$ alone, hence this "jump data" needs to be appended to the semimartingale $X$. Note that such "jump data" will typically have to be defined on an extended probability space and will be adapted to an extended filtration $\left(\mathcal{G}_{t}\right)_{t \in \mathbb{R}_{+}}$to which $X$ will still be adapted. 
In order to record the necessary "jump data", fix a holonomy bundle $P(u)$ with a holonomy group $\operatorname{Hol}(u)$ and consider some (global) section $q: M \rightarrow P(u)$, i.e. a map $q$ satisfying $\pi \circ q=\mathrm{id}_{M}$. We require that $q$ is measurable and that $q$ maps any compact set in $M$ into a pre-compact set in $P(u)$. These requirements would hold trivially if $q$ were smooth, but in general it is not possible to construct even a continuous section. However, the fibre bundle structure allows us to find smooth local sections. Combining countably many such local sections we can always find a measurable (global) section $q$ satisfying compactness condition. Fixing one such section allows us to record the "jump data" as an $\mathbb{R}^{d}$-valued process.

Definition 2.17. An $\mathbb{R}^{d}$-valued process $J$ An $\mathbb{R}^{d}$-valued process $J$ (possibly) on an extended probability space and adapted to filtration $\left(\mathcal{G}_{t}\right)_{t \in \mathbb{R}_{+}}$is said to represent the jumps of a semimartingale $X$ on $[0, \tau)$ if

$$
X_{s}=\operatorname{Exp}_{X_{s-}}\left(q\left(X_{s-}\right) J_{s}\right) \text { for } s<\tau \quad \text { and } \sum_{u \leq t}\left|J_{u}\right|^{2}<\infty \text { for } t \in \mathbb{R}_{+},
$$

for a measurable global section $q: M \rightarrow P(u)$ mapping compact sets in $M$ into precompact sets in $P(u)$.

Remark 2.18. (i) It would suffice for a process $J$ to be defined only on $[0, \tau)$, but it turns out to be convenient in proofs to have it defined for all times in $[0, \infty)$.

(ii) If we start with an anti-development $W$ and use it to construct the processes $U$ and $X$, we may then represent the jumps of $X$ by a process $J_{s}:=q\left(X_{s-}\right)^{-1} U_{s-} \Delta W_{s}$. Processes $U$ and $X$ are càdlàg, hence locally bounded, meaning that for every $t \in \mathbb{R}_{+}$ and a.e. $\omega$ the paths $X(\omega)$ and $U(\omega)$ are included in compact sets for all times in $[0, t]$, hence by compactness condition on $q$ also matrices $q\left(X_{s-}(\omega)\right)^{-1} U_{s-}(\omega)$ are included in a compact set for $s \in[0, t]$, so that these matrices are bounded in operator norm. Since jumps of a semimartingale $W$ are square summable we thus immediately obtain the summability condition in (2.10). Hence it is natural to require this summability condition for any process $J$ in Definition 2.17. Note also that any such $J$ may be different from 0 for at most countably many times $s$.

(iii) For a general semimartingale $X$ there might not exist any process $J$ which represents its jumps. However, (ii) above shows that if $X$ has an anti-development, such a process always exists. Actually, Theorem 2.19 below shows that being able to represent jumps of $X$ by some process $J$ is equivalent to $X$ having a stochastic anti-development. The property of $X$ having a stochastic anti-development does not depend on the choice of a section. Moreover, this means that if a pair $(q, J)$ satisfies (2.10), then for any other measurable section $\widetilde{q}$ satisfying compactness condition we can define a process $\widetilde{J}_{s}:=\widetilde{q}\left(X_{s-}\right)^{-1} U_{s-} \Delta W_{s}$ (where $U$ and $W$ are as in (ii)) so that $(\widetilde{q}, \widetilde{J})$ satisfies (2.10), including the summability condition. This shows that for a semimartingale $X$ the property of being able to represent its jumps as in Definition 2.17 does not depend on the choice of a measurable section satisfying compactness condition. Furthermore, the results of Theorem 2.19 can be obtained for any choice of such section.

When constructing a horizontal lift $U$ and anti-development $W$ of $X=\left(X_{t}\right)_{t \in[0, \tau)}$, the process $J$ will enable us to uniquely determine the corresponding jumps of $U$ and $W$. More precisely, by Definition 2.17, Remark 2.18 and equation (A.3) we should have

$$
U_{s}=\exp \left(H_{U_{s-}^{-1} q\left(X_{s-}\right) J_{s}}\right)\left(U_{s-}\right)
$$

and 


$$
\Delta W_{s}=U_{s-}^{-1} q\left(X_{s-}\right) J_{s}
$$

for all (jump) times $s<\tau$.

Thus, we are able to start with $X$ and $J$ and construct a unique horizontal lift and anti-development.

Theorem 2.19. Let $X$ be a $M$-valued semimartingale defined on $[0, \tau)$ along with a process $J$ which represents its jumps. Let $u_{0} \in \pi^{-1}\left(\left\{X_{0}\right\}\right) \cap P(u)$. Then there exists a unique horizontal lift $U=\left(U_{t}\right)_{t \in[0, \tau)}$ of $X$ with $U_{0}=u_{0}$ and values in $P(u)$ satisfying (2.11). It has a unique anti-development $W=\left(W_{t}\right)_{t \in[0, \tau)}$ satisfying (2.12).

Remark 2.20. Both $U$ and $W$ are adapted to filtration $\left(\mathcal{G}_{t}\right)_{t \in \mathbb{R}_{+}}$to which $X$ and $J$ were adapted to start with.

As a corollary we may recover the results of [32, Thm. 3.2 and Prop. 4.3]. When there exists a unique geodesic between any two endpoints of a jump of a semimartingale $X$, then there exists a unique ${ }^{2}$ process $J$ which represents its jumps and hence we obtain a unique horizontal lift and a unique anti-development.

\section{Examples}

In this section we show how Definition 2.8 relates to previous definitions of Lévy processes on Riemannian manifolds and on Lie groups. We also provide some concrete examples which illustrate different definitions and (slight) differences between them.

\subsection{Riemannian manifolds}

On a Riemannian manifold $M$ there exists a canonical Levi-Civita connection uniquely determined by the metric. It is easy to see that the associated fundamental horizontal vector fields are then also vector fields on the orthonormal frame bundle $O(M)$ which consists of orthonormal frames $u: \mathbb{R}^{d} \rightarrow T_{\pi(u)} M$, i.e. frames which are isometries. Thus, the horizontal Lévy process - a solution of a Marcus SDE (2.4) - stays on the orthonormal frame bundle if it is started there. In [3] they consider such horizontal Lévy processes and then their projections to $M$ are Markov if the integrator $Y$ is a Lévy process invariant by the full orthogonal group $\mathrm{O}(d)$. In this case they call the projections isotropic Lévy processes on the Riemannian manifold $M$.

However, it is often possible to reduce the orthonormal frame bundle even further, and hence require less invariance. In the Riemannian case such a reduction was first considered in [30, Sec. 3]. Namely, compatibility of the Levi-Civita connection with the metric means that if $u$ is some (any) fixed orthonormal frame, then the holonomy bundle $P(u)$ is a subbundle of $O(M)$ and the holonomy group $\operatorname{Hol}(u)$ is a Lie subgroup of $\mathrm{O}(d)$. Thus, for a fixed orthonormal frame $u$ the Lévy processes from Definition 2.8 require less invariance of the integrator than isotropic Lévy processes, and hence the latter are just a special case of the former.

Nevertheless, there are still examples where using a holonomy bundle reduction does not extend the class of processes. For a simple example we may consider a $d$-dimensional sphere $\mathbb{S}^{d}:=\left\{x \in \mathbb{R}^{d+1} ;|x|=1\right\}$ equipped with a standard round metric, i.e. the metric inherited by the standard Euclidean metric of the ambient space $\mathbb{R}^{d+1}$. Then it is not hard to see that the holonomy group is the special orthogonal group $\mathrm{SO}(d)$, hence isotropic Lévy processes exhaust all Lévy processes on a sphere.

Still, holonomy bundle reduction often extends the class of Lévy process and to see the difference between only isotropic Lévy processes and all possible Lévy processes

\footnotetext{
${ }^{2}$ Process $J$ is uniquely determined only on $[0, \tau)$, but those are the only values used in construction of horizontal lift and anti-development in Theorem 2.19
} 
from Definition 2.8 we now analyse a couple of examples on flat Riemannian manifolds (meaning the metric is locally isometric to the standard Euclidean one) where isotropic Lévy processes form a strictly smaller class of processes than all the possible processes from Definition 2.8.

\subsubsection{Tori}

Let $n \in \mathbb{N}$ and consider the action of a discrete group $\mathbb{Z}^{n}$ on $\mathbb{R}^{n}$ given by

$$
\varphi: \mathbb{R}^{n} \times \mathbb{Z}^{n} \rightarrow \mathbb{R}^{n}, \varphi(x, z)=x+z .
$$

The action is free and proper so the Quotient manifold theorem [27, Thm. 21.10] implies that the quotient $\mathbb{T}^{n}:=\mathbb{R}^{n} / \mathbb{Z}^{n}$ has a unique smooth structure making it into a smooth manifold of dimension $n$, such that the quotient projection $\pi: \mathbb{R}^{n} \rightarrow \mathbb{T}^{n}$ is a smooth submersion. The smooth manifold $\mathbb{T}^{n}$ is known as an $n$-dimensional torus. Furthermore, the projection $\pi$ is a local diffeomorphism and $\mathbb{R}^{n}$ is a universal covering space of $\mathbb{T}^{n}$. We consider a standard Euclidean metric $g$ on $\mathbb{R}^{n}$ and since $\varphi$ acts by isometries, there is a canonical metric $\widetilde{g}$ on $\mathbb{T}^{n}$ defined by

$$
\widetilde{g}_{p}(u, v):=g_{x}\left(\left(d \pi_{x}\right)^{-1} u,\left(d \pi_{x}\right)^{-1} v\right),
$$

where $x \in \mathbb{R}^{n}$ is any point with $\pi(x)=p \in \mathbb{T}^{n}$ and $u, v \in T_{p} \mathbb{T}^{n}$.

We now compute the holonomy group. Let $\widetilde{\gamma}$ be a loop based at $p \in \mathbb{T}^{n}$. Covering space theory tells us that for any $x \in \pi^{-1}(p)$ there exists a unique curve $\gamma$ in $\mathbb{R}^{n}$ with $\gamma_{0}=x$, such that $\pi \circ \gamma=\widetilde{\gamma}$. The endpoint of $\gamma$ is not necessarily equal to $x$, but it is always some element of the fibre $\pi^{-1}(p)$, so it can be written as $\varphi(x, z)$ for a unique $z \in \mathbb{Z}^{n}$. It is now clear that the parallel transport $\widetilde{\tau}_{\tilde{\gamma}}$ along $\widetilde{\gamma}$ of tangent vectors in $T_{p} \mathbb{T}^{n}$ can be expressed in term of parallel transport $\tau_{\gamma}$ along $\gamma$ in $\mathbb{R}^{n}$ and it is given by

$$
\widetilde{\tau}_{\widetilde{\gamma}}=d \pi_{\varphi(x, z)} \circ \tau_{\gamma} \circ\left(d \pi_{x}\right)^{-1}=d \pi_{x} \circ\left(d \varphi(\cdot, z)_{x}\right)^{-1} \circ \tau_{\gamma} \circ\left(d \pi_{x}\right)^{-1}=\operatorname{id}_{T_{p} \mathbb{T}^{n}} .
$$

In the last equality we used that the parallel transport of a flat Euclidean metric, when we identify $T \mathbb{R}^{n}$ by $\mathbb{R}^{n} \times \mathbb{R}^{n}$, is given exactly by the translation $\varphi(\cdot, z)$. Hence, the holonomy group is trivial and the holonomy bundle is diffeomorphic to $\mathbb{T}^{n}$. It also follows that each horizontal vector field $H_{i}$ on the holonomy bundle is $\pi$-related (see (4.2) below for the definition) to the standard vector field $\partial_{i}$ on $\mathbb{R}^{d}$ for $i=1, \ldots, d$. This implies the geodesic completeness of the torus and shows that every Lévy process on $\mathbb{T}^{n}$ is given as $\pi(X)$, where $X$ is an arbitrary Lévy process on $\mathbb{R}^{n}$. On the other hand isotropic Lévy processes on $\mathbb{T}^{n}$ are exactly those given as $\pi(X)$ for an isotropic Lévy process $X$ on $\mathbb{R}^{n}$ and thus form a strictly smaller class. Actually, $\mathbb{Z}^{n}$ is a closed subgroup of $\mathbb{R}^{n}$ and the action we considered is given via the group operation, so torus $\mathbb{T}^{n}$ can actually be given a structure of a Lie group and we can alternatively proceed as in Section 3.2 below to recover the same results.

\subsubsection{Generalized Klein bottles}

The second example generalizes the Klein bottle. Let $n \in \mathbb{N}$ and let $\mathbb{R}^{n+1}=\mathbb{R}^{n} \times \mathbb{R}$. The group we consider is a semi-direct product $\mathbb{Z}^{n} \rtimes \mathbb{Z}$, so that the group multiplication is given by $(a, b) \cdot(z, w):=\left((-1)^{w} a+z, b+w\right)$. Note that this is not a subgroup of $\mathbb{R}^{n+1}$ with group operation of addition. The group action we consider is

$$
\varphi:\left(\mathbb{R}^{n} \times \mathbb{R}\right) \times\left(\mathbb{Z}^{n} \rtimes \mathbb{Z}\right) \rightarrow \mathbb{R}^{n} \times \mathbb{R}, \varphi((x, y),(z, w))=\left((-1)^{w} x+z, y+w\right) .
$$

Again, the action is free and proper so the quotient $\mathbb{K}^{n+1}:=\left(\mathbb{R}^{n} \times \mathbb{R}\right) /\left(\mathbb{Z}^{n} \rtimes \mathbb{Z}\right)$ has a unique smooth structure making it into a smooth manifold of dimension $n$. We call the 
manifold $\mathbb{K}^{n+1}$ an $(n+1)$-dimensional Klein bottle. The projection $\pi: \mathbb{R}^{n+1} \rightarrow \mathbb{K}^{n+1}$ is a local diffeomorphism and $\mathbb{R}^{n+1}$ is a universal covering space of $\mathbb{K}^{n+1}$. As in (3.1) we can endow $\mathbb{K}^{n+1}$ with a flat Riemannian metric turning in into a geodesically complete manifold.

To compute the holonomy group we proceed as above. Let $\widetilde{\gamma}$ be a loop based at $p \in \mathbb{K}^{n+1}$ and $\gamma$ a unique curve in $\mathbb{R}^{n+1}$ starting at $(x, y) \in \pi^{-1}(p)$, such that $\pi \circ \gamma=\widetilde{\gamma}$. The endpoint of $\gamma$ can be written as $\varphi((x, y),(z, w))$ for a unique element $(z, w) \in \mathbb{Z}^{n} \rtimes \mathbb{Z}$. Again, we may compute the parallel transport as

$$
\widetilde{\tau}_{\widetilde{\gamma}}=d \pi_{\varphi((x, y),(z, w))} \circ \tau_{\gamma} \circ\left(d \pi_{(x, y)}\right)^{-1}=d \pi_{(x, y)} \circ\left(d \varphi(\cdot,(z, w))_{(x, y)}\right)^{-1} \circ \tau_{\gamma} \circ\left(d \pi_{(x, y)}\right)^{-1} .
$$

We then easily see that the map $\left(d \varphi(\cdot,(z, w))_{(x, y)}\right)^{-1} \circ \tau_{\gamma}$ can be expressed in terms of standard coordinates on $\mathbb{R}^{n+1}$ as

$$
\left[\begin{array}{cc}
(-1)^{w} I_{n} & 0 \\
0^{\top} & 1
\end{array}\right]
$$

where $I_{n}$ is an identity matrix of dimension $n$ and $0 \in \mathbb{R}^{n}$ is a zero column vector. Thus, the holonomy group is isomorphic to $\mathbb{Z}_{2}$ and all the Lévy processes on $\mathbb{K}^{n+1}$ are given by $\pi(X)$, where $X$ is a Lévy process on $\mathbb{R}^{n+1}$ with law invariant under the multiplication by the matrix

$$
\left[\begin{array}{cc}
-I_{n} & 0 \\
0^{\top} & 1
\end{array}\right]
$$

Again, this is less restrictive then only using isotropic Lévy processes on $\mathbb{R}^{n+1}$ which induce isotropic Lévy processes on $\mathbb{K}^{n+1}$

\subsection{Lie groups}

We will use Definition 2.8 to construct Lévy processes on a connected $d$-dimensional Lie group $G$ and compare them to a classical definition of (left) Lévy processes on Lie groups defined via independent and stationary increments (see [28] or [19]).

First, we need to select a suitable connection on $G$. Lie groups are parallelizable since they possess a global frame. To get a global frame we consider a basis $V_{1}, \ldots, V_{d}$ of a Lie algebra $\mathfrak{g}:=T_{e} G$ of $G$, where $e$ denotes the identity element. Given an element $V \in \mathfrak{g}$ we define a left-invariant vector field $V^{L}$ by $\left.V^{L}\right|_{g}:=d\left(L_{g}\right)_{e} V$, where $d\left(L_{g}\right)_{e}$ is a differential of left multiplication $L_{g}: h \mapsto g h$. Then a global frame is given by $V_{1}^{L}, \ldots, V_{d}^{L}$. Analogously, we could define right-invariant vector fields $V_{1}^{R}, \ldots, V_{d}^{R}$, which also form a global frame. Having a global frame allows us to define a connection (covariant derivative) on elements of the global frame and then extending it to all vector fields by $C^{\infty}(G)$-linearity in the first argument and by the product rule in the second argument.

We want to select an appropriate connection so that the parallel transport induced by any curve $\gamma$ is given exactly by the differential of the left multiplication $d\left(L_{\gamma_{1} \gamma_{0}^{-1}}\right)_{\gamma_{0}}$ where the left multiplication takes the initial point $\gamma_{0}$ of the the curve to its end point $\gamma_{1}$. Since we want that this equality holds for any curve $\gamma$, we quickly see that the only possible such connection is given via

$$
\nabla_{V_{i}^{L}} V_{j}^{L}=0 \text { for all } i, j=1, \ldots, d
$$

and we call this connection a left-invariant connection. It is easily seen that this connection turns $G$ into a geodesically complete manifold. If we wanted that the parallel transport of a connection is given by the differential of right translations, we could use the same prescription, but the global frame would have to consist of right-invariant vector fields $V_{1}^{R}, \ldots, V_{d}^{R}$. 
It is obvious that the holonomy group associated with the left-invariant connection is trivial, so for any frame $u \in F(G)$ the restricted projection $\pi: P(u) \rightarrow G$ is actually a diffeomorphism between a holonomy bundle $P(u)$ and a Lie group $G$. For definiteness, we fix $u$ to be the frame with $\pi(u)=e$ for which $u\left(e_{i}\right)=V_{i}$ holds for every $i=1, \ldots, d$.

In order to get a Lévy process $X$ on the Lie group $G$ we first construct a horizontal Lévy process $U$ on $P(u)$ as a solution of a Marcus SDE (2.4), where $H_{i}$ are fundamental horizontal vector fields of the left-invariant connection and then $X=\pi(U)$. Since $\pi: P(u) \rightarrow G$ is a diffeomorphism, Lemma 4.3 shows that the process $X$ is actually a solution of a Marcus SDE

$$
X_{t}=\pi_{*} H_{i}\left(X_{t-}\right) \diamond \mathrm{d} Y_{t}^{i},
$$

and due to our particular choice of frame $u$ and the associated holonomy bundle $P(u)$, the push-forward vector fields $\pi_{*} H_{i}$ are equal to $V_{i}^{L}$ for each $i=1, \ldots, d$, so $X$ is a solution of a Marcus SDE

$$
\mathrm{d} X_{t}=V_{i}^{L}\left(X_{t-}\right) \diamond \mathrm{d} Y_{t}^{i} .
$$

Since $V_{i}^{L}$ are left-invariant vector fields and since $Y$ is a Lévy process it seems obvious the process $X$ has independent and stationary increments, making it a (left) Lévy process on a Lie group $G$ as per classical definition. However, we would also like to relate some characteristics of the Lévy process $Y$ to those of the process $X$. In particular, we would like to relate their Lévy measures, which govern their jumps and are therefore a measures on $\mathbb{R}^{d} \backslash\{0\}$ and $G \backslash\{e\}$, respectively, which satisfy certain integrability conditions.

Process $X$ is a solution of (3.2) and by the definition of the Marcus SDE when $Y$ jumps at time $t$, then $X$ also jumps at the same time and $X_{t}=X_{t-} \exp \left(V_{i} \Delta Y_{t}^{i}\right)$, where $\exp : \mathfrak{g} \rightarrow G$ is an exponential map of a Lie group. Lie algebra $\mathfrak{g}$ is isomorphic to $\mathbb{R}^{d}$ so given a Lévy measure $\nu$ of $Y$ we can construct a push-forward measure $\widetilde{\mu}:=\widetilde{\exp }_{*} \nu$ on $G$ where $\widetilde{\exp }: \mathbb{R}^{d} \ni x=x^{i} e_{i} \mapsto \exp \left(x^{i} V_{i}\right) \in G$ and then the restricted measure $\mu:=\left.\widetilde{\mu}\right|_{G \backslash\{e\}}$ should be a Lévy measure of the process $X$. We summarize these facts in the next proposition and provide the proof in Section 4.5.

Proposition 3.1. Let $X$ be a solution of a Marcus SDE (3.2), where $Y$ is an $\mathbb{R}^{d}$-valued Lévy process with a Lévy measure $\nu$. Then $X$ is a (left) Lévy process on $G$ with a Lévy measure $\mu:=\left.\widetilde{\exp }_{*} \nu\right|_{G \backslash\{e\}}$.

Proposition 3.1 shows that every process $X$ constructed via Definition 2.8 is a classical (left) Lévy process in $G$, but the converse need not be true. Being able to represent a Lévy process $X$ in $G$ as a solution of a Marcus SDE (3.2) is equivalent to an existence of a stochastic horizontal lift $U$ of $X$, such that its anti-development $Y$ is a Euclidean Lévy process. By Proposition 3.1 we know that Lévy measures of processes $X$ and $Y$ then need to be related by $\mu=\left.\widetilde{\exp }_{*} \nu\right|_{G \backslash\{e\}}$. But the exponential map need not be surjective, so there can exist Lévy measures on $G$, and hence (left) Lévy processes on $G$, which cannot be represented by this construction. Obviously, to even have a chance of finding an anti-development $Y$, we must be able to represent all the jumps of $X$ via the exponential map. Actually, as soon as this is the case, we are able to construct the process $Y$.

Proposition 3.2. Let $X$ be a left Lévy process in $G$ with a Lévy measure $\mu$. Then there exists an $\mathbb{R}^{d}$-valued Lévy process $Y$ such that $X$ satisfies Marcus SDE (3.2) if and only if $\mu\left(\operatorname{Im}(\exp )^{c}\right)=0$.

The proof of the proposition is given in Section 4.5 and we can also deduce the following corollary.

Corollary 3.3. Let $G$ be a Lie group with a surjective exponential map. Then a process $X$ is a (left) Lévy process in $G$ if and only if it is a solution of a Marcus SDE (3.2) for some $\mathbb{R}^{d}$-valued Lévy process $Y$. 
Lévy processes on smooth manifolds with a connection

Remark 3.4. (i) A statement analogous to the if part of Corollary 3.3 is stated in the review article [5, p. 8]. In fact, Proposition 3.1 shows that the if part holds without the assumption on the surjectivity of the exponential map.

(ii) In the only if part, for a process $X$ to satisfy Marcus SDE (3.2), one needs to construct a Lévy process $Y$ on the extended probability space such that (3.2) holds. It is not sufficient to construct a Lévy process $Y$ on some other unrelated probability space, such that a different solution $\widetilde{X}$ of (3.2) driven by $Y$ has the same law as $X$. The construction of the Lévy process $Y$ from $X$ is given in the proof of our main result, Theorem 2.14.

Conditions of the corollary often hold, for example if the group is connected and compact or if it is simply connected and nilpotent. On the other hand, we trivially observe non-surjectivity of the exponential map when a Lie group is not connected, since then the image of the (connected) Lie algebra under the (continuous) exponential map obviously cannot be the whole (disconnected) group. A non-trivial example of a connected (and non-compact) Lie group with a non-surjective exponential map is given by the group $\mathrm{GL}(2)_{+}$, i.e. the group of matrices in $\mathbb{R}^{2} \otimes \mathbb{R}^{2}$ with a strictly positive determinant.

\subsection{Further examples}

Definition 2.8 allows us to define Lévy processes on an arbitrary smooth manifold as soon as we specify a connection on it. On a general smooth manifold there are (infinitely) many possible choices of a connection and in some cases, as is seen in previous sections, there is a canonical choice. The particular connection we use is intimately related to the Definition 2.8 and leads to often non-trivial conditions (2.6). We will now show how these conditions restrict and may sometimes even severely limit the class of Lévy processes on a manifold.

Recall that the holonomy group $\operatorname{Hol}(u)$ of the holonomy bundle $P(u)$ (associated with a frame $u \in F(M)$ ) is a Lie subgroup of the general linear group GL $(d)$, where $d$ is the dimension of the underlying manifold $M$. The holonomy group is determined by the connection and it is natural to consider the question of whether any Lie subgroup of the general linear group GL $(d)$ can be realized as a holonomy group of some manifold with the connection. A partial answer has been known for over half a century and [17] shows that for any connected Lie subgroup of GL $(d)$ there exist some manifold with a connection, such that the induced holonomy group is exactly the selected subgroup.

Hence it is possible to find a manifold with a connection, such that its holonomy group is equal to $\mathrm{GL}(d)_{+}$, i.e. invertible matrices with a strictly positive determinant. In this case the Lévy integrator in the definition of a horizontal Lévy process has to satisfy conditions (2.6) and it is quickly seen that having such a large holonomy group forces the Lévy integrator to be a trivial process constantly equal to 0 . Hence all Lévy processes on such a manifold have to be constant as well. This is quite an extreme example, but it shows that the choice of the connection can have a profound effect on the size of the class of Lévy processes.

For a less extreme example let us consider a group consisting of matrices of the form

$$
\left[\begin{array}{cc}
I_{d-1} & 0 \\
0^{\top} & \beta
\end{array}\right]
$$

where $I_{d-1}$ is an identity matrix of dimension $d-1,0 \in \mathbb{R}^{d-1}$ is a zero column vector and $\beta>0$. Such matrices for strictly positive values of parameter $\beta$ form a connected Lie subgroup of $\mathrm{GL}(d)$, hence it may once again be realized as a holonomy group of some manifold with a connection. In this case conditions (2.6) force the Lévy integrator to stay in the $(d-1)$-dimensional hyperplane perpendicular to $e_{d}$. There are no further 
restrictions, so we may take any Lévy integrator, which is given as an arbitrary $(d-1)$ dimensional Euclidean Lévy process in the first $d-1$ components and with vanishing last component. This essentially means that the Lévy process on the manifold "loses one direction" in which it may move, but this is a geometric restriction required to keep our definition well-posed.

\subsubsection{Cylinder with a family of connections}

We now present an example of a 2-dimensional manifold with a 1-parameter family of connections on it. Depending on the value of the parameter, the holonomy group turns out to be a cyclic subgroup of $\mathrm{SO}(2)$, which is either finite or an infinite dense subgroup isomorphic to $\mathbb{Z}$. In particular, if the rank of the holonomy group is even, it contains the element $-I_{2}$, providing a class of examples where we may simplify the jump part of the generator of Lévy processes on the manifold as in Remark 2.13(i). Moreover, the connection introduced below is not torsion-free (for $\alpha \neq 0$ ) and thus cannot be the Levi-Civita connection of a Riemannian manifold.

The 2-dimensional manifold $M=\mathbb{S}^{1} \times \mathbb{R}$ is paralellizable with a global frame given by the vector fields $V_{\theta}, V_{z}$ defined as follows: under the natural inclusion $\mathbb{S}^{1} \times \mathbb{R} \rightarrow \mathbb{R}^{2} \times \mathbb{R}$ let $V_{\theta}=\left.\left(-y \frac{\partial}{\partial x}+x \frac{\partial}{\partial y}\right)\right|_{M}$ and $V_{z}=\left.\left(\frac{\partial}{\partial z}\right)\right|_{M}$. We can now specify a connection (covariant derivative) via the global frame:

$$
\nabla_{V_{z}} V_{\theta}=\nabla_{V_{z}} V_{z}=0, \quad \nabla_{V_{\theta}} V_{\theta}=-\alpha V_{z}, \quad \nabla_{V_{\theta}} V_{z}=\alpha V_{\theta},
$$

where $\alpha \in \mathbb{R}$ is a real parameter. By using (A.1) we can bound the derivatives of geodesics (in the ambient space) which shows that $M$ equipped with this connection is geodesically complete. Furthermore, it is easy to describe the parallel transport of such a connection as we may use a global frame to identify different tangent spaces. If we are moving along $V_{z}$, the tangent vectors remain unchanged, while moving along $V_{\theta}$ rotates the tangent vectors by an angle expressed in radians as $\alpha$ times the directed length of the horizontal displacement. Hence the holonomy group is generated by the rotation around the origin through angle $2 \pi \alpha$. The holonomy group is thus isomorphic to $\mathbb{Z}$ if $\alpha$ is irrational and to $\left(\mathbb{Z}_{q},+\right)$ (for some $q \in \mathbb{N}$ ) if $\alpha$ is a rational. In particular, by taking $\alpha=\frac{1}{2}$, the holonomy group equals $\left\{I_{2},-I_{2}\right\}$.

\section{Proofs}

\subsection{Marcus SDEs on manifolds}

We start with the proof of our main existence and uniqueness result for Marcus SDEs.

Proof of Proposition 2.3. In [25] Marcus SDE was studied in Euclidean spaces. They use a component-wise definition of a solution; however, the chain rule [25, Prop. 4.2] shows that it is equivalent to Definition 2.1 when the smooth manifold $M$ is taken to be the Euclidean space and the vector fields are Lipschitz continuous, so that the solution is defined for all times in $\mathbb{R}_{+}$. To prove the proposition for a general smooth manifold $M$, we use Whitney's embedding theorem to embed $M$ as a closed submanifold of the Euclidean space $\mathbb{R}^{n}$ for a sufficiently large ${ }^{3} n$. In particular, this allows us to identify the point $\partial$ of compactification $\widehat{M}$ with the point $\infty$ of compactification of $\mathbb{R}^{d}$. We can then smoothly extend vector fields $V_{i}$ on $M$ to vector fields $V_{i}^{*}$ on the whole $\mathbb{R}^{n}$. Being smooth, these vector fields are locally Lipschitz continuous. We consider closed balls $\mathbb{B}_{k}:=\left\{x \in \mathbb{R}^{n} ;|x| \leq k\right\}$ for $k \in \mathbb{N}$ and for each $k \in \mathbb{N}$ we take smooth functions $\varphi_{k}$ such that $\varphi_{k}=1$ on $\mathbb{B}_{k}$ and $\operatorname{supp} \varphi_{k} \subseteq \mathbb{B}_{k+1}$. By denoting $V_{i}^{(k)}:=\varphi_{k} V_{i}^{*}$ for $i=1, \ldots, \ell$ we

\footnotetext{
${ }^{3}$ Twice the dimension of the manifold $M$ suffices.
} 
see that vector fields $V_{i}^{(k)}$ are compactly supported, hence they are globally Lipschitz continuous and any linear combination of them is a complete vector field.

We consider a Marcus SDE

$$
\mathrm{d} X_{t}^{(k)}=V_{i}^{(k)}\left(X_{t-}^{(k)}\right) \diamond \mathrm{d} W_{t}^{i}
$$

on $\mathbb{R}^{n}$ with the initial condition $X_{0}^{(k)} \in M$ and without loss of generality (by translation) we may assume that $X_{0}^{(k)}=0 \in \mathbb{B}_{k}$. Then [25, Thm. 3.2] shows that there exists a unique solution $\left(X_{t}^{(k)}\right)_{t \in \mathbb{R}_{+}}$of (4.1) and since vector fields $V_{i}^{(k)}$ are tangent to $M$, [25, Prop 4.2] shows that the solution $X^{(k)}$ stays on $M$. We define

$$
\tau_{k}:=\inf \left\{s \in \mathbb{R}_{+} ; \exists \theta \in[0,1] \text { such that } \exp \left(\theta V_{i} \Delta W_{s}^{i}\right)\left(X_{s-}^{(k)}\right) \notin \mathbb{B}_{k}\right\},
$$

i.e. $\tau_{k}$ is the first time the process $X^{(k)}$ or the "hidden" trajectory of its jump exits $\mathbb{B}_{k}$. For $k \leq \ell$ consider the processes $X^{(k)}$ and $X^{(\ell)}$. Restricted to the interval $\left[0, \tau_{k}\right)$, they depend on their corresponding vector fields $V_{i}^{(k)}$ and $V_{i}^{(\ell)}$ restricted to $\mathbb{B}_{n}$, but the vector fields coincide there. Therefore, uniqueness of solutions from [25, Thm. 3.2] means that the processes $X^{(k)}$ and $X^{(\ell)}$ coincide on $\left[0, \tau_{k}\right)$, so we may unambiguously define a process $\left(X_{t}\right)_{t \in[0, \tau)}$ where $\tau=\lim _{k \rightarrow \infty} \tau_{k}$ so that $X_{t}=X_{t}^{(k)}$ on $\left[0, \tau_{k}\right)$. It easily follows that the process $X$ is exactly the unique solution of Marcus SDE (2.1) on $[0, \tau)$. Finally, we have to prove that $\tau$ is an explosion time and that $\left\{\tau_{n} ; n \in \mathbb{N}\right\}$ form its announcing sequence. This immediately follows once we show that $\tau_{n}<\tau$ a.s. for every $n \in \mathbb{N}$.

In general, it is possible that $X^{(k)}$ exits $\mathbb{B}_{k}$ at a time $\tau_{k}$ by a jump - such a jump being induced by the jump $\Delta W_{\tau_{k}}$ of the integrator. If the vector field $V_{i} \Delta W_{\tau_{k}}^{i}$ were not complete then $\exp \left(V_{i} \Delta W_{\tau_{k}}^{i}\right)\left(X_{\tau_{k}-}\right)$ might not be defined and in this case $\tau_{\ell}=\tau_{k}$ for all $\ell \geq k$, and hence $\tau_{k}=\tau$. However, this cannot happen if as assumed all the vector fields $a^{i} V_{i}$ are complete, since then the flow exponential map is always defined, thus the norm of $\exp \left(V_{i} \Delta W_{\tau_{k}}^{i}\right)\left(X_{\tau_{k}-}\right)$ is smaller than $L$ for some $L \in \mathbb{N}$ larger than $k$ and we have $\tau_{k}<\tau_{L} \leq \tau$ for any $k \in \mathbb{N}$ and this finishes the proof.

We now prove a couple of technical lemmas which elucidate the transformation rules of Marcus SDEs. First, we may consider a Marcus SDE and linearly transform the vector fields appearing in the SDE. Next lemma shows that this is the same as appropriately linearly transforming the integrator.

Lemma 4.1. Let $\ell, m \in \mathbb{N}$ and let $V_{1}, \ldots, V_{\ell} \in \Gamma(T M)$ be smooth vector fields on $M$ and $W$ be an $\mathbb{R}^{m}$-valued càdlàg semimartingale. Suppose that $\widetilde{V}_{1}, \ldots, \widetilde{V}_{m} \in \Gamma(T M)$ are smooth vector fields given as $\widetilde{V}_{i}:=A_{i}^{j} V_{j}$ for some matrix $A \in \mathbb{R}^{\ell} \otimes \mathbb{R}^{m}$. Then an $M$-valued semimartingale $X$ defined on $[0, \tau)$ is a solution of Marcus $S D E$

$$
\mathrm{d} X_{t}=\widetilde{V}_{i}\left(X_{t-}\right) \diamond \mathrm{d} W_{t}^{i},
$$

on $[0, \tau)$ if and only if it is a solution of Marcus $S D E$

$$
\mathrm{d} X_{t}=V_{j}\left(X_{t-}\right) \diamond \mathrm{d} \widetilde{W}_{t}^{j}
$$

on $[0, \tau)$, where an $\mathbb{R}^{\ell}$-valued semimartingale $\widetilde{W}$ is given by $\widetilde{W}:=A W$.

Proof. The defining property (2.2) for the first Marcus SDE is the equation

$$
\begin{aligned}
f\left(X_{t}\right)-f\left(X_{0}\right) & =\int_{0}^{t} A_{i}^{j} V_{j} f\left(X_{s-}\right) \circ \mathrm{d} W_{s}^{i} \\
& +\sum_{0<s \leq t}\left(f\left(\exp \left(A_{i}^{j} V_{j} \Delta W_{s}^{i}\right)\left(X_{s-}\right)\right)-f\left(X_{s-}\right)-A_{i}^{j} V_{j} f\left(X_{s-}\right) \Delta W_{s}^{i}\right)
\end{aligned}
$$


for any $f \in C^{\infty}(M)$, and simple algebra using $\widetilde{W}^{j}=A_{i}^{j} W^{i}$ shows that this is also exactly the defining property for the second Marcus SDE.

Another important property of the Marcus SDE is that it behaves nicely under diffeomorphisms. We will first prove a slightly more general result. Let $\Phi: M \rightarrow N$ be a smooth map. We say that vector fields $V \in \Gamma(T M)$ and $V^{\prime} \in \Gamma(T N)$ are $\Phi$-related if

$$
d \Phi_{p} V_{p}=V_{\Phi(p)}^{\prime}
$$

for every $p \in M$, where $d \Phi_{p}$ represents the differential of smooth map $\Phi$ at a point $p$.

Lemma 4.2. Let $M$ and $N$ be smooth manifolds and let $\Phi: M \rightarrow N$ be a smooth map. Let $V_{1}, \ldots, V_{\ell} \in \Gamma(T M)$ be vector fields and let semimartingale $X$ defined on $[0, \tau)$ be an $M$-valued solution of a Marcus SDE

$$
\mathrm{d} X_{t}=V_{i}\left(X_{t-}\right) \diamond \mathrm{d} W_{t}^{i}
$$

on $[0, \tau)$. Suppose that $V_{1}^{\prime}, \ldots, V_{\ell}^{\prime} \in \Gamma(T N)$ are vector fields, such that $V_{i}$ and $V_{i}^{\prime}$ are $\Phi$-related for every $i=1, \ldots, \ell$. Then the $N$-valued semimartingale $\widetilde{X}:=\Phi(X)$ is a solution of a Marcus SDE

$$
\mathrm{d} \widetilde{X}_{t}=V_{i}^{\prime}\left(\widetilde{X}_{t-}\right) \diamond \mathrm{d} W_{t}^{i}
$$

on $[0, \tau)$.

Proof. We need to show that the defining property (2.2) of Marcus SDE (4.4) holds for $\widetilde{X}$ when we take an arbitrary smooth function $f \in C^{\infty}(N)$. We can take a function $f \circ \Phi \in C^{\infty}(M)$ and use the defining property (2.2) of Marcus SDE (4.3) for $X$ to get

$$
\begin{aligned}
f\left(\widetilde{X}_{t}\right)-f\left(\widetilde{X}_{0}\right) & =\int_{0}^{t} V_{i}(f \circ \Phi)\left(X_{s-}\right) \circ \mathrm{d} W_{s}^{i} \\
& +\sum_{0<s \leq t}\left(f \circ \Phi\left(\exp \left(V_{i} \Delta W_{s}^{i}\right)\left(X_{s-}\right)\right)-f\left(\widetilde{X}_{s-}\right)-V_{i}(f \circ \Phi)\left(X_{s-}\right) \Delta W_{s}^{i}\right) .
\end{aligned}
$$

Equation (4.2) implies that $V_{i}^{\prime} f(\Phi(p))=V_{i}(f \circ \Phi)(p)$ holds for any $f \in C^{\infty}(N)$ and $p \in M$, so we have

$$
V_{i}^{\prime} f\left(\widetilde{X}_{t-}\right)=V_{i}(f \circ \Phi)\left(X_{t-}\right)
$$

for any $i=1, \ldots, \ell$.

Using linearity in (4.2) shows that vector fields $V_{x}:=x^{i} V_{i}$ and $V_{x}^{\prime}:=x^{i} V_{i}^{\prime}$ are $\Phi$ related for any $x=x^{i} e_{i} \in \mathbb{R}^{d}$. By (4.2), if a tangent vector of a curve $\gamma$ is equal to $\left.V_{x}\right|_{p}$ at $p$, then the tangent vector of a curve $\Phi \circ \gamma$ is equal to $\left.V_{x}^{\prime}\right|_{\Phi(p)}$ at $\Phi(p)$. In particular, $\Phi$ maps integral curves of $V_{x}$ to integral curves of $V_{x}^{\prime}$, hence

$$
\Phi\left(\exp \left(V_{x}\right)(p)\right)=\exp \left(V_{x}^{\prime}\right)(\Phi(p))
$$

holds for all $p \in M$.

Inserting (4.6) and (4.7) into equation (4.5) yields exactly the defining property for Marcus SDE (4.4) and $\widetilde{X}$ is its solution.

When a map $\Phi: M \rightarrow N$ is a diffeomorphism, we can define a push-forward $\Phi_{*}$ : $\Gamma(T M) \rightarrow \Gamma(T N)$ so that for a vector field $V \in \Gamma(T M)$ the push-forward vector field $\Phi_{*} V \in \Gamma(T N)$ is defined by the prescription

$$
\left(\Phi_{*} V\right)_{q}:=d \Phi_{\Phi^{-1}(q)} V_{\Phi^{-1}(q)}
$$

for any $q \in N$. It is clear from this definition that the vector fields $V$ and $\Phi_{*} V$ are $\Phi$-related, so the following lemma is an immediate corollary of Lemma 4.2. 
Lévy processes on smooth manifolds with a connection

Lemma 4.3. Let $\Phi: M \rightarrow N$ be a diffeomorphism and let semimartingale $X$ defined on $[0, \tau)$ be an $M$-valued solution of a Marcus SDE

$$
\mathrm{d} X_{t}=V_{i}\left(X_{t-}\right) \diamond \mathrm{d} W_{t}^{i}
$$

on $[0, \tau)$. Then the $N$-valued semimartingale $\widetilde{X}:=\Phi(X)$ is a solution of a Marcus SDE

$$
\mathrm{d} \widetilde{X}_{t}=\Phi_{*} V_{i}\left(\widetilde{X}_{t-}\right) \diamond \mathrm{d} W_{t}^{i}
$$

on $[0, \tau)$.

\subsection{Proofs of Proposition 2.9, Lemma 2.11 and Proposition 2.12}

Proof of Proposition 2.9. In the discussion prior to the Definition 2.8 it was already shown that the invariance of the integrator under the holonomy group implies Markovianity of the projected process. We now show that changing the frame and the associated holonomy bundle does not alter the class of Lévy processes on a connected smooth manifold $M$.

Consider a different frame $v \in F(M)$. Connectedness of $M$ implies that $\pi: P(v) \rightarrow M$ is surjective and if $v^{\prime} \in P(v)$ is another frame, then $P\left(v^{\prime}\right)=P(v)$ and $\operatorname{Hol}\left(v^{\prime}\right)=\operatorname{Hol}(v)$, hence we may assume that $v=u g$ for some $g \in \operatorname{GL}(d)$ so that $\operatorname{Hol}(v)=g^{-1} \operatorname{Hol}(u) g$ holds [23, Prop. II.4.1]. We take a Lévy process $X=\pi(U)$, where $U$ is a horizontal Lévy process and a solution of a Marcus SDE (2.4) with a $\operatorname{Hol}(u)$-invariant integrator $Y$ and $U_{0} \in P(u)$. Hence, if we denote $\widetilde{U}:=U g$ and $\widetilde{Y}:=g^{-1} Y$, then Lemmas 4.3 , A.2 and 4.1 imply that $\widetilde{U}$ is a $P(v)$-valued solution of the Marcus SDE

$$
\mathrm{d} \widetilde{U}_{t}=H_{i}\left(\widetilde{U}_{t-}\right) \diamond \mathrm{d} \widetilde{Y}_{t}^{i}
$$

with an integrator $\widetilde{Y}$, which is $\operatorname{Hol}(v)$-invariant. Since $X=\pi(U)=\pi(\widetilde{U}), X$ is also a Lévy process when defined through the holonomy bundle $P(v)$. This shows that the same processes are called Lévy processes on $M$ no matter which holonomy bundle we use for their definition.

Proof of Lemma 2.11. It is a well-known fact that a Euclidean Lévy process $Y$ with the generating triplet $(a, \nu, b)$ can be expressed via the Lévy-Itô decomposition [2, Thm. 2.4.16]

$$
Y_{t}^{i}=b^{i} t+\sigma_{j}^{i} B_{t}^{j}+\int_{0}^{t} \int_{0<|x|<1} x^{i} \widetilde{N}(\mathrm{~d} s, \mathrm{~d} x)+\int_{0}^{t} \int_{|x| \geq 1} x^{i} N(\mathrm{~d} s, \mathrm{~d} x),
$$

where $\sum_{k=1}^{d} \sigma_{k}^{i} \sigma_{k}^{j}=a^{i j}, B$ is a standard $d$-dimensional Brownian motion, $N$ is an independent Poisson random measure on $\mathbb{R}_{+} \times \mathbb{R}^{d}$ with mean measure $\mathcal{L} e b \otimes \nu$, i.e. $\mathbb{E}[N([0, t], A)]=t \nu(A)$, and $\widetilde{N}$ is a compensated random measure defined by $\widetilde{N}([0, t], A)=$ $N([0, t], A)-t \nu(A)$. Since $U$ is a solution of Marcus SDE (2.4), we can take $f \in C^{\infty}(P(u))$ and substitute (4.9) into the defining property of Marcus SDEs to get

$$
\begin{aligned}
f\left(U_{t}\right) & =f\left(U_{0}\right)+b^{i} \int_{0}^{t} H_{i} f\left(U_{s-}\right) \mathrm{d} s+\sigma_{j}^{i} \int_{0}^{t} H_{i} f\left(U_{s-}\right) \circ \mathrm{d} B_{s}^{j} \\
& +\int_{0}^{t} \int_{0<|x|<1} x^{i} H_{i} f\left(U_{s-}\right) \widetilde{N}(\mathrm{~d} s, \mathrm{~d} x)+\int_{0}^{t} \int_{|x| \geq 1} x^{i} H_{i} f\left(U_{s-}\right) N(\mathrm{~d} s, \mathrm{~d} x) \\
& +\int_{0}^{t} \int_{\mathbb{R}^{d} \backslash\{0\}}\left(f\left(\exp \left(H_{x}\right)\left(U_{s-}\right)\right)-f\left(U_{s-}\right)-x^{i} H_{i} f\left(U_{s-}\right)\right) N(\mathrm{~d} s, \mathrm{~d} x) .
\end{aligned}
$$


After recalling the definition of Stratonovich integral and some algebraic manipulation using $\widetilde{N}(\mathrm{~d} s, \mathrm{~d} x)=N(\mathrm{~d} s, \mathrm{~d} x)-\mathrm{d} s \nu(\mathrm{d} x)$ we can rewrite the equation as

$$
\begin{aligned}
f\left(U_{t}\right) & =f\left(U_{0}\right)+\sigma_{j}^{i} \int_{0}^{t} H_{i} f\left(U_{s-}\right) \mathrm{d} B_{s}^{j}+\int_{0}^{t} \int_{\mathbb{R}^{d} \backslash\{0\}}\left(f\left(\exp \left(H_{x}\right)\left(U_{s-}\right)\right)-f\left(U_{s-}\right)\right) \tilde{N}(\mathrm{~d} s, \mathrm{~d} x) \\
& +\int_{0}^{t} \mathscr{L}_{U}(f)\left(U_{s}\right) \mathrm{d} s,
\end{aligned}
$$

where $\mathscr{L}_{U}$ is given by (2.5). Since the first two integrals in the right hand side of equation (4.10) are (local) martingales, standard arguments show that $\mathscr{L}_{U}$ is the (extended) generator (see $[13,9]$ ) of the horizontal Lévy process $U$, at least on smooth functions.

Proof of Proposition 2.12. Since $f(X)=f \circ \pi(U)$, the only possible prescription for the generator of $X$ is given by

$$
\mathscr{L}_{X}(f)(p)=\mathscr{L}_{U}(f \circ \pi)(v),
$$

where $v$ is any frame in $P(u)$ with $\pi(v)=p$ and $\mathscr{L}_{U}$ is a generator of a horizontal Lévy process obtained in Lemma 2.11. We use invariance conditions (2.6) to apply [13, Thm. 10.13] from which it follows that right hand side of (4.11) is indeed well-defined and equal to the generator of $X$. The theorem also identifies the domain of the generator.

In order to finish the proof and get the explicit expression (2.7) for the generator we simply use in the above expression the equality (A.3) relating flow and geodesic exponential maps.

\subsection{Proof of Theorem 2.19}

For the proof of the theorem we will need the following estimate for the flow exponential map of the fundamental horizontal vector fields in local coordinates on the frame bundle.

Lemma 4.4. Consider local coordinates on $O \subseteq M$ and the associated local coordinates on $\pi^{-1}(O) \subseteq F(M)$. Let $u=\left(x^{i}, r_{m}^{k}\right)=(x, r) \in \pi^{-1}(O), c=c^{n} e_{n} \in \mathbb{R}^{d}$ and consider $\exp \left(H_{c}\right)(u)=\psi(1)$, where $\psi(t)=\psi(t, u, c)$ is the integral curve of $H_{c}$ starting at $\psi(0)=u$, which we assume stays in $\pi^{-1}(O)$ for all $t \in[0,1]$. Then we have

$$
\exp \left(H_{c}\right)(u)=\left(x^{i}, r_{m}^{k}\right)+\left(c^{n} r_{n}^{i},-c^{n} r_{n}^{j} r_{m}^{l} \Gamma_{j l}^{k}(x)\right)+c^{n} c^{n^{\prime}} F_{n n^{\prime}}(\psi(\theta))
$$

for some smooth functions $F_{n n^{\prime}}$ with values in $\mathbb{R}^{d+d^{2}}$ and some $\theta \in(0,1)$.

Proof. Via local coordinates we may consider $\psi$ as a smooth curve in $\mathbb{R}^{d+d^{2}}$ and we can use Taylor approximation up to order 2 to write:

$$
\exp \left(H_{c}\right)(u)=\psi(1)=\psi(0)+\dot{\psi}(0)+\frac{1}{2} \ddot{\psi}(\theta)
$$

for some $\theta \in(0,1)$. We obviously have $\psi(0)=u=\left(x^{i}, r_{m}^{k}\right)$. Since $\psi$ is an integral curve of a fundamental horizontal vector field, we use the representation in local coordinates from Lemma A.1 to write

$$
\dot{\psi}(t)=H_{c}(\psi(t))=\left(c^{n} \psi_{n}^{i}(t),-c^{n} \psi_{n}^{j}(t) \psi_{m}^{l}(t) \Gamma_{j l}^{k}(\pi \circ \psi(t))\right),
$$

from which $\dot{\psi}(0)=\left(c^{n} r_{n}^{i},-c^{n} r_{n}^{j} r_{m}^{l} \Gamma_{j l}^{k}(x)\right)$ easily follows. Hence, we have already obtained the first two terms in (4.12) and we only need to obtain the final one. We compute 
Lévy processes on smooth manifolds with a connection

further time derivative of (4.13) in which we can use (4.13) once more to get

$$
\begin{aligned}
\ddot{\psi}^{i}(t) & =c^{n} \dot{\psi}_{n}^{i}(t)=-c^{n} c^{n^{\prime}} \psi_{n^{\prime}}^{j}(t) \psi_{n}^{l}(t) \Gamma_{j l}^{i}(\pi \circ \psi(t)) \\
\ddot{\psi}_{m}^{k}(t) & =c^{n} c^{n^{\prime}} \psi_{n^{\prime}}^{\alpha}(t) \psi_{n}^{\beta}(t) \Gamma_{\alpha \beta}^{j}(\pi \circ \psi(t)) \psi_{m}^{l}(t) \Gamma_{j l}^{k}(\pi \circ \psi(t)) \\
& +c^{n} c^{n^{\prime}} \psi_{n}^{j}(t) \psi_{n^{\prime}}^{\alpha}(t) \psi_{m}^{\beta}(t) \Gamma_{\alpha \beta}^{l}(\pi \circ \psi(t)) \Gamma_{j l}^{k}(\pi \circ \psi(t)) \\
& -c^{n} c^{n^{\prime}} \psi_{n}^{j}(t) \psi_{m}^{l}(t) \partial_{\alpha} \Gamma_{j l}^{k}(\pi \circ \psi(t)) \psi_{n^{\prime}}^{\alpha}(t),
\end{aligned}
$$

so we can indeed write $\frac{1}{2} \ddot{\psi}(\theta)=c^{n} c^{n^{\prime}} F_{n n^{\prime}}(\psi(\theta))$ for some smooth functions $F_{n n^{\prime}}$, and thus establish the results of the lemma.

Proof of Theorem 2.19. We will construct a horizontal lift and an anti-development using local charts and then patch the local solutions together. We consider local coordinates $\varphi^{\alpha}$ on a precompact set $O \subseteq M$ and the associated local coordinates $\phi^{\alpha}, \phi_{m}^{k}$ on $\pi^{-1}(O) \subseteq$ $F(M)$. Inspired by the deterministic horizontal lift equation (A.2) and condition (2.11) we claim that the horizontal lift $U=\left(\varphi^{i}(X), R_{m}^{k}\right)$ solves the following SDE written in local coordinates:

$$
\begin{aligned}
R_{m}^{k}(t) & =R_{m}^{k}(0)-\int_{0}^{t} R_{m}^{l}(s-) \Gamma_{j l}^{k}\left(X_{s-}\right) \circ \mathrm{d} \varphi^{j}\left(X_{s}\right) \\
& +\sum_{0<s \leq t}\left(\exp \left(H_{U_{s-}^{-1} q\left(X_{s-}\right) J_{s}}\right)\left(U_{s-}\right)_{m}^{k}-R_{m}^{k}(s-)+R_{m}^{l}(s-) \Gamma_{j l}^{k}\left(X_{s-}\right) \Delta \varphi^{j}\left(X_{s}\right)\right),
\end{aligned}
$$

for every $t<\widetilde{\tau}$, where $\widetilde{\tau}=\inf \left\{s \in \mathbb{R}_{+} ; X_{s} \notin O\right\}$. Its anti-development $W$ is then defined by

$$
W_{t}^{i}=\int_{0}^{t} \bar{R}_{j}^{i}(s-) \circ \mathrm{d} \varphi^{j}\left(X_{s}\right)+\sum_{0<s \leq t}\left(\left(U_{s-}^{-1} q\left(X_{s-}\right) J_{s}\right)^{i}-\bar{R}_{j}^{i}(s-) \Delta \varphi^{j}\left(X_{s}\right)\right)
$$

for $t<\widetilde{\tau}$, where $\bar{R}$ is an inverse of $R$, i.e. $\bar{R}_{j}^{i} R_{l}^{k}=\delta_{l}^{i} \delta_{j}^{k}$. Note that such $U$ and $W$ will be adapted to $\left(\mathcal{G}_{t}\right)_{t \in \mathbb{R}_{+}}$.

We first analyse the equality (4.14). For it to even make sense, we need to show that the sum on the right hand side is a.s. finite. We assume that we already have such a process $U$ which is càdlàg, hence $U_{-}$is locally bounded and we denote its trajectory up to time $t$ by $\kappa_{t}(\omega)$, which is a bounded set a.s. Hence there exists $\varepsilon(\omega)>0$ and an a.s. bounded set $D_{t}(\omega) \subseteq \pi^{-1}(O)$ such that every integral curve of a horizontal vector fields $H_{c}$ with initial point in $\kappa_{t}$ and $|c|<\varepsilon$ stays $^{4}$ in $D_{t}$ up to time 1 . We denote $c_{s}=U_{s-}^{-1} q\left(X_{s-}\right) J_{s}$, where $U_{s-}^{-1} q\left(X_{s-}\right)$ is a locally bounded holonomy group valued process. This local boundedness together with condition (2.11) implies that there are at most finitely many times $s \in[0, t]$ with $\left|c_{s}\right| \geq \varepsilon$, so the sum of absolute values of terms corresponding to such times is a finite random variable which we denote by $C_{t}$. Since $X_{s-}=\pi\left(U_{s-}\right)$ and $X_{s}=\pi\left(\exp \left(H_{c_{s}}\right)\left(U_{s-}\right)\right)$, we can use Lemma 4.4 and write each of the remaining terms corresponding to $\left|c_{s}\right|<\varepsilon$ as

$$
\begin{aligned}
& R_{m}^{k}(s-)-c_{s}^{n} R_{n}^{j}(s-) R_{m}^{l}(s-) \Gamma_{j l}^{k}\left(X_{s-}\right)+c_{s}^{n} c_{s}^{n^{\prime}}\left(F_{n n^{\prime}}\right)_{m}^{k}\left(\psi\left(\theta, U_{s-}\right)\right)-R_{m}^{k}(s-) \\
+ & R_{m}^{l}(s-) \Gamma_{j l}^{k}\left(X_{s-}\right)\left(\varphi^{j}\left(X_{s-}\right)+c_{s}^{n} R_{n}^{j}(s-)+c_{s}^{n} c_{s}^{n^{\prime}}\left(F_{n n^{\prime}}\right)^{j}\left(\psi\left(\theta, U_{s-}\right)\right)-\varphi^{j}\left(X_{s-}\right)\right) \\
= & c_{s}^{n} c_{s}^{n^{\prime}}\left(\widetilde{F}_{n n^{\prime}}\left(\psi\left(\theta, U_{s-}\right)\right)\right)_{m}^{k},
\end{aligned}
$$

\footnotetext{
${ }^{4}$ This follows from results about systems of ODEs in Euclidean setting and uses only that the set of initial points is bounded and that the vector field is locally Lipschitz in an argument as well as in parameter.
} 
for some smooth functions $\widetilde{F}_{n n^{\prime}}$ and some $\theta=\theta(s, \omega) \in(0,1)$. Triangle and CauchySchwarz inequalities then imply that the absolute value of the whole sum is smaller or equal to

$$
\begin{aligned}
& C_{t}+\sum_{0<s \leq t,\left|c_{s}\right|<\varepsilon}\left|c_{s}^{n} c_{s}^{n^{\prime}}\left(\widetilde{F}_{n n^{\prime}}\left(\psi\left(\theta, U_{s-}\right)\right)\right)_{m}^{k}\right| \\
\leq & C_{t}+d^{-1} \sup _{1 \leq n, n^{\prime} \leq d, \theta \in[0,1], s \in[0, t],\left|c_{s}\right|<\varepsilon}\left|\left(\widetilde{F}_{n n^{\prime}}\left(\psi\left(\theta, U_{s-}\right)\right)\right)_{m}^{k}\right| \sum_{0<s \leq t,\left|c_{s}\right|<\varepsilon}\left|c_{s}\right|^{2} \\
\leq & C_{t}+K_{t} L_{t}^{2} d^{-1} \sum_{s \leq t}\left|J_{s}\right|^{2}<\infty,
\end{aligned}
$$

where $L_{t}$ is the (local) bound for the holonomy group valued process $U_{s-}^{-1} q\left(X_{s-}\right)$ and we have used that the final sum is finite by (2.10). We obtain the bound $K_{t}(\omega)$ for the supremum as a consequence of taking a supremum of finitely many continuous functions over a subset of a bounded set $D_{t}$.

Next, we need to show that there exists a solution of SDE (4.14) which is a semimartingale. We wish to use the general theory of SDEs against semimartingales, so we need to rewrite the equation into a correct form. We start by considering SDE (4.14) in the matrix form so that the first integral can be written as

$$
\int_{0}^{t} F(R(s-)) \circ \mathrm{d} \varphi\left(X_{s}\right)=\int_{0}^{t} F_{j}(R(s-)) \circ \mathrm{d} \varphi^{j}\left(X_{s}\right),
$$

where the random coefficients $F_{j}:\left(\mathbb{R}^{d} \otimes \mathbb{R}^{d}\right) \times \Omega \rightarrow \mathbb{R}^{d} \otimes \mathbb{R}^{d}$ are given by

$$
F_{j}(r, \omega)_{m j}^{k}:=r_{m}^{l} \Gamma_{j l}^{k}\left(X_{s-}(\omega)\right)
$$

for $j=1, \ldots, d$ and $\varphi(X)$ is an $\mathbb{R}^{d}$-valued semimartingale $\varphi(X):=\left(\varphi^{1}(X), \ldots, \varphi^{d}(X)\right)^{\top}$. Note that as a function of $r$, the coefficient $F$ is linear. Next, we need to appropriately transform the sum. We view the sum as an $\mathbb{R}^{d} \otimes \mathbb{R}^{d}$-valued processes and we may use Lemma A. 3 to express the sum as a component-wise integral

$$
\int_{0}^{t} A\left(X_{s-}, J_{s}\right) R(s-) \mathrm{d} Z_{s}
$$

where $Z_{t}=\sum_{0<s<t}\left|J_{s}\right|^{2}$ is a (finite variation) real semimartingale. The $\mathbb{R}^{d} \otimes \mathbb{R}^{d}$-valued process $A\left(X_{t-}, J_{t}\right)$ has components given by

$$
A\left(X_{t-}, J_{t}\right)_{m}^{k}:=\left(\exp \left(H_{\widetilde{U}_{t-}^{-1} q\left(X_{t-}\right) J_{t}}\right)\left(\widetilde{U}_{t-}\right)_{m}^{k}-\delta_{m}^{k}+B\left(X_{t-}, J_{t}\right)_{m}^{k}\right) /\left|J_{t}\right|^{2},
$$

where $\delta_{m}^{k}=\mathbb{1}(k=m)$ is Dirac delta, the process $\widetilde{U}$ is given by $\widetilde{U}:=U R^{-1}$, and the $\mathbb{R}^{d} \otimes \mathbb{R}^{d}$-valued process $B\left(X_{t-}, J_{t}\right)$ has components given by

$$
B\left(X_{t-}, J_{t}\right)_{m}^{k}:=\Gamma_{j m}^{k}\left(X_{t-}\right) \Delta \varphi^{j}\left(X_{t}\right)=\Gamma_{j m}^{k}\left(X_{t-}\right)\left(\varphi^{j}\left(\operatorname{Exp}_{X_{t-}}\left(q\left(X_{t-}\right) J_{t}\right)\right)-\varphi^{j}\left(X_{t-}\right)\right) .
$$

Notice that $\widetilde{U}$ has a simple representation in local coordinates as $\widetilde{U}_{t}=\left(X_{t}, I_{d}\right)$, so that $A\left(X_{t-}, J_{t}\right)$ is indeed just a function of $X_{t-}$ and $J_{t}$ and there is no dependence on the process $R$. Therefore, we can rewrite SDE (4.14) in matrix form as

$$
R(t)=R(0)+\int_{0}^{t} F(R(s-)) \circ \mathrm{d} \varphi\left(X_{s}\right)+\int_{0}^{t} A\left(X_{s-}, J_{s}\right) R(s-) \mathrm{d} Z_{s} .
$$

Note that the integrands in (4.16) are linear in $R(s-)$ with random linear coefficients given by $\Gamma_{j l}^{k}\left(X_{s-}\right)$ and $A\left(X_{s-}, J_{s}\right)$. Thus (4.16) is a standard form of an SDE against 
semimartingales with the integrands being linear, hence functional Lipschitz (in the terminology of [33, Ch. V]). More precisely, the integrands are obtained via an action of a functional Lipschitz operator on the process $R$. Functional Lipschitzness is a consequence of linearity and a.s. boundedness of linear coefficients on finite intervals. Indeed, for a.e. $\omega$ and every $t>0$ we have $\sup _{s \leq t}\left|\Gamma_{j l}^{k}\left(X_{s-}(\omega)\right)\right|<\infty$, which follows from the càdlàg property of $X$ and the smoothness of Christoffel symbols. Moreover, it also holds that $\sup _{s \leq t}\left|A\left(X_{s-}(\omega), J_{s}(\omega)\right)\right|<\infty$. Observe first that $A$ is non-zero only when $J$ is non-zero, which holds for at most countably many times, and further for every $\varepsilon>0$ we have $\left|J_{s}(\omega)\right|>\varepsilon$ for at most finitely times $s$. Thus, the finiteness of the supremum holds trivially at finitely many large-jump times. We may assume that all of the (possibly infinitely many) remaining small jumps are bounded by a constant $\varepsilon$. By the boundedness of $\widetilde{U}_{s-}^{-1}(\omega) q\left(X_{s-}(\omega)\right)$ by some constant $K$ (see Remark 2.18 (ii)) we can therefore consider $\varepsilon$ small enough, such that the transformed jumps $\widetilde{U}_{s-}^{-1}(\omega) q\left(X_{s-}(\omega)\right) J_{s}(\omega)$, which are bounded by $\varepsilon K$, are small enough, so that we may apply Lemma 4.4 with $c=\widetilde{U}_{s-}^{-1}(\omega) q\left(X_{s-}(\omega)\right) J_{s}(\omega)$ and $u=\widetilde{U}_{s-}(\omega)=\left(x^{j}, r_{m}^{k}\right)=\left(\varphi^{j}\left(X_{s-}(\omega)\right), \delta_{m}^{k}\right)$. Using (4.12) we compute

$$
\begin{aligned}
A\left(X_{s-}, J_{s}\right)_{m}^{k} & =\left(\delta_{m}^{k}-c^{j} \Gamma_{j m}^{k}\left(X_{s-}(\omega)\right)+c^{n} c^{n^{\prime}} F_{n n^{\prime}}(\psi(\theta))_{m}^{k}-\delta_{m}^{k}\right) /\left|J_{s}(\omega)\right|^{2} \\
& +\Gamma_{j m}^{k}\left(X_{s-}(\omega)\right)\left(\varphi^{j}\left(X_{s-}(\omega)\right)+c^{j}+c^{n} c^{n^{\prime}} F_{n n^{\prime}}(\psi(\theta))^{j}-\varphi^{j}\left(X_{s-}(\omega)\right)\right) /\left|J_{s}(\omega)\right|^{2} \\
& =c^{n} c^{n^{\prime}}\left(F_{n n^{\prime}}(\psi(\theta))_{m}^{k}+\Gamma_{j m}^{k}\left(X_{s-}(\omega)\right) F_{n n^{\prime}}(\psi(\theta))^{j}\right) /\left|J_{s}(\omega)\right|^{2} \\
& \leq K^{2} \sum_{n, n^{\prime}=1}^{d}\left(F_{n n^{\prime}}(\psi(\theta))_{m}^{k}+\Gamma_{j m}^{k}\left(X_{s-}(\omega)\right) F_{n n^{\prime}}(\psi(\theta))^{j}\right),
\end{aligned}
$$

from which boundedness of supremum of $A$ follows by using the càdlàg property of $X$, smoothness of maps $\Gamma_{j m}^{k}$ and $F_{n n^{\prime}}$, and the fact that integral curves $\psi$ stay inside a compact set due to small sizes of jumps and the càdlàg property of $X$.

Thus, the coefficients of the SDE are indeed functional Lipschitz and [33, Thm. 7 in Ch. V] implies that there exists a unique solution $R$ of the SDE (4.16) and the linearity of coefficients implies non-explosion of the solution, i.e. process $R$ is defined until the stopping time $\widetilde{\tau}$. Actually, there is a potential issue as the coefficient $A\left(X_{s-}, J_{s}\right) R(s-)$ is not predictable, but it is still optionally measurable w.r.t. $\left(\mathcal{G}_{t}\right)_{t \in \mathbb{R}_{+}}$and the integral is against an increasing finite variation process so we may still use a trivial extension of [33, Thm. 7 in Ch. V] (see also remarks in the proof of [25, Thm. 3.2]).

We claim that the frame bundle valued process $U$ given in local coordinates as $U=(X, R)$ is exactly the horizontal lift of $X$. It is clear that $U$ is a lift of $X$, i.e. it projects to $X$, but we still need to check that it is horizontal, more precisely, we need to identify its anti-development. The candidate for the anti-development is given by equation (4.15), initially only for $t<\widetilde{\tau}$, and similar calculation as above shows that the sum in (4.15) is almost surely convergent so that $W$ is a semimartingale. We need to check that $W$ is an anti-development of $U$, i.e. we need to show that $U$ solves Marcus SDE (2.1) when the integrator $W$ is given by (4.15). It is enough to check the defining property of Marcus $\operatorname{SDE}(2.1)$ for functions which constitute a local chart on $\pi^{-1}(O)$.

We first take $f=\phi^{\alpha}$, so that $\phi^{\alpha}\left(U_{t}\right)=\varphi^{\alpha}\left(X_{t}\right)$ and note that by the representation in local coordinates of fundamental horizontal vector fields from Lemma A.1 we have 


$$
\begin{aligned}
& H_{i} \phi^{\alpha}(u)=r_{i}^{\alpha} \text { for } u=\left(x^{j}, r_{m}^{k}\right) \text {. Therefore, } \\
& \quad \int_{0}^{t} H_{i} \phi^{\alpha}\left(U_{s-}\right) \circ \mathrm{d} W_{s}^{i}+\sum_{0<s \leq t}\left(\phi^{\alpha}\left(\exp \left(H_{i} \Delta W_{s}^{i}\right)\left(U_{s-}\right)\right)-\phi^{\alpha}\left(U_{s-}\right)-H_{i} \phi^{\alpha}\left(U_{s-}\right) \Delta W_{s}^{i}\right) \\
& \quad=\int_{0}^{t} R_{i}^{\alpha}(s-) \bar{R}_{j}^{i}(s-) \circ \mathrm{d} \varphi^{j}\left(X_{s}\right) \\
& \quad+\sum_{0<s \leq t}\left(R_{i}^{\alpha}(s-)\left(U_{s-}^{-1} q\left(X_{s-}\right) J_{s}\right)^{i}-R_{i}^{\alpha}(s-) \bar{R}_{j}^{i}(s-) \Delta \varphi^{j}\left(X_{s}\right)\right) \\
& +\sum_{0<s \leq t}\left(\varphi^{\alpha}\left(X_{s}\right)-\varphi^{\alpha}\left(X_{s-}\right)-R_{i}^{\alpha}(s-) \Delta W_{s}^{i}\right) \\
& =\int_{0}^{t} 1 \circ \mathrm{d} \varphi^{\alpha}\left(X_{s}\right)+\sum_{0<s \leq t}\left(R_{i}^{\alpha}(s-) \Delta W_{s}^{i}-\Delta \varphi^{\alpha}\left(X_{s}\right)+\Delta \varphi^{\alpha}\left(X_{s}\right)-R_{i}^{\alpha}(s-) \Delta W_{s}^{i}\right) \\
& =\varphi^{\alpha}\left(X_{s}\right)-\varphi^{\alpha}\left(X_{0}\right)=\phi^{\alpha}\left(U_{t}\right)-\phi^{\alpha}\left(U_{0}\right)
\end{aligned}
$$

holds, where we used (4.15) in the first equality and (2.12) in the second inequality. This establishes the defining property of Marcus SDE (2.1) for $f=\phi^{\alpha}$.

We now take $f=\phi_{m}^{k}$, so that $\phi_{m}^{k}\left(U_{s}\right)=R_{m}^{k}(s)$ and note that Lemma A.1 implies the equality $H_{i} \phi_{m}^{k}(u)=-r_{i}^{\beta} r_{m}^{l} \Gamma_{\beta l}^{k}(p)$ for $u=\left(p^{i}, r_{m}^{k}\right)$. Then

$$
\begin{aligned}
& \left.\int_{0}^{t} H_{i} \phi_{m}^{k}\left(U_{s-}\right) \circ \mathrm{d} W_{s}^{i}+\sum_{0<s \leq t}\left(\phi_{m}^{k}\left(\exp \left(H_{i} \Delta W_{s}^{i}\right)\left(U_{s-}\right)\right)-\phi_{m}^{k}\left(U_{s-}\right)-H_{i} \phi_{m}^{k}\left(U_{s-}\right) \Delta W_{s}^{i}\right)\right) \\
& =-\int_{0}^{t} R_{i}^{\beta}(s-) R_{m}^{l}(s-) \Gamma_{\beta l}^{k}\left(X_{s-}\right) \bar{R}_{j}^{i}(s-) \circ \mathrm{d} \varphi^{j}\left(X_{s}\right) \\
& +\sum_{0<s \leq t}\left(-R_{i}^{\beta}(s-) R_{m}^{l}(s-) \Gamma_{\beta l}^{k}\left(X_{s-}\right) \Delta W_{s}^{i}+R_{i}^{\beta}(s-) R_{m}^{l}(s-) \Gamma_{\beta l}^{k}\left(X_{s-}\right) \bar{R}_{j}^{i}(s-) \Delta \varphi^{j}\left(X_{s}\right)\right) \\
& \left.+\sum_{0<s \leq t}\left(\phi_{m}^{k}\left(\exp \left(H_{U_{s-}^{-1} q\left(X_{s-}\right) J_{s}}\right)\left(U_{s-}\right)\right)-\phi_{m}^{k}\left(U_{s-}\right)+R_{i}^{\beta}(s-) R_{m}^{l}(s-) \Gamma_{\beta l}^{k}\left(X_{s-}\right) \Delta W_{s}^{i}\right)\right) \\
& =-\int_{0}^{t} R_{m}^{l}(s-) \Gamma_{j l}^{k}\left(X_{s-}\right) \circ \mathrm{d} \varphi^{j}\left(X_{s}\right) \\
& +\sum_{0<s \leq t}\left(\phi_{m}^{k}\left(\exp \left(H_{U_{s-}^{-1} q\left(X_{s-}\right) J_{s}}\right)\left(U_{s-}\right)\right)-\phi_{m}^{k}\left(U_{s-}\right)+R_{m}^{l}(s-) \Gamma_{j l}^{k}\left(X_{s-}\right) \Delta \varphi^{j}\left(X_{s}\right)\right) \\
& =R_{m}^{k}(t)-R_{m}^{k}(0)=\phi_{m}^{k}\left(U_{t}\right)-\phi_{m}^{k}\left(U_{0}\right)
\end{aligned}
$$

holds, where we used (4.15) in the first equality and (4.14) in the penultimate equality and this establishes the defining property of Marcus SDE (2.1) for $f=\phi_{m}^{k}$. Thus, we have established that $W$ is an anti-development of $U$ (and $X$ ), hence $U$ is indeed a horizontal process and a horizontal lift of $X$.

We want to extend processes $U$ and $W$ to a larger time interval. Note that we can find a (random) sequence of precompact charts $O_{n}$ such that $X_{\tau_{n-1}} \in O_{n}$ for $n \in \mathbb{N}$ where $\tau_{0}=0$ and $\tau_{n}=\inf \left\{s>\tau_{n-1} ; X_{s} \notin O_{n}\right\}$ for $n \in \mathbb{N}$ and such that $\tau_{n} \rightarrow \tau$ (the lifetime of $X$ ) as $n \rightarrow \infty$. Construction from above first defines horizontal lift and anti-development on $\left[0, \tau_{1}\right)$. At time $\tau_{1}$ we then define $U_{\tau_{1}}=\exp \left(H_{U_{\tau_{1}-}^{-1} q\left(X_{\tau_{1}-}\right) J_{\tau_{1}}}\right)\left(U_{\tau_{1}-}\right)$ and $W_{\tau_{1}}=W_{\tau_{1}-}+U_{\tau_{1}-}^{-1} q\left(X_{\tau_{1}-}\right) J_{\tau_{1}}$ and this makes $U$ horizontal lift and $W$ an antidevelopment of $X$ on $\left[0, \tau_{1}\right]$. Since $U_{\tau_{1}} \in \pi^{-1}\left(O_{2}\right)$ we can reuse the construction from above, where now the initial point is $U_{\tau_{1}}$ and this defines processes $U$ and $W$ also on $\left[\tau_{1}, \tau_{2}\right)$. Iteratively, this defines the horizontal lift and anti-development on $\left[0, \tau_{n}\right)$ so by sending $n \rightarrow \infty$, the horizontal lift and anti-development are semimartingales defined until the lifetime of $X$. 
We still need to prove the uniqueness of horizontal lift and anti-development and hence independence of selection of local charts used in the construction. We will proceed similarly to [32, Thm. 3.2]. Suppose that $U, W$ are a horizontal lift and anti-development, respectively, induced by $X, J$ and suppose $V, \widetilde{W}$ are another such pair with $U_{0}=V_{0}$. Since $\pi(U)=\pi(V)=X$, there is a unique càdlàg process $g_{s}$ with values in $\mathrm{GL}(d)$ such that $V_{s}=U_{s} g_{s}=\rho\left(U_{s}, g_{s}\right)$, where $\rho: F(M) \times \mathrm{GL}(d) \rightarrow M$ is a right group action of GL $(d)$ on the frame bundle. In particular, $g_{0}=e$ and the equality

$$
\Delta \widetilde{W}_{t}=V_{t-}^{-1} q\left(X_{t-}\right) J_{t}=g_{t-}^{-1} U_{t-}^{-1} q\left(X_{t-}\right) J_{t}=g_{t-}^{-1} \Delta W_{t}
$$

holds. Actually, it is not hard to see that

$$
\widetilde{W}_{t}=\int_{0}^{t} g_{s}^{-1} \circ \mathrm{d} W_{t}
$$

holds. Since $W$ (resp. $\widetilde{W}$ ) is an anti-development of $U$ (resp. $V$ ), we can use the appropriate versions of (2.11) to compute

$$
U_{t} g_{t}=V_{t}=\exp \left(H_{\Delta \widetilde{W}_{t}}\right)\left(V_{t-}\right)=\exp \left(H_{g_{t-}^{-1} \Delta W_{t}}\right)\left(U_{t-} g_{t-}\right)=\exp \left(H_{\Delta W_{t}}\right)\left(U_{t-}\right) g_{t-}=U_{t} g_{t-},
$$

where we used Lemma A.3 in the penultimate equality. It follows that $g_{t}=g_{t-}$ and hence the process $g_{t}$ is continuous.

To prove uniqueness it is equivalent to show that the process $g_{t}$ from above is constantly equal to the identity. We consider local coordinates $u^{\beta}$ on $F(M)$ (these are all $\phi^{\alpha}$ and $\phi_{m}^{k}$ from above combined) and local coordinates $h^{k}$ on $\operatorname{GL}(d)$. For ease of notation we denote $V_{t}^{\alpha}=u^{\alpha}\left(V_{t}\right), U_{t}^{\beta}=u^{\beta}\left(U_{t}\right)$ and $g_{t}^{k}=h^{k}\left(g_{t}\right)$. By the defining property of Marcus SDEs we have

$$
\begin{aligned}
V_{t}^{\alpha} & =V_{0}^{\alpha}+\int_{0}^{t} H_{i} u^{\alpha}\left(V_{s-}\right) \circ \mathrm{d} \widetilde{W}_{s}^{i} \\
& +\sum_{0<s \leq t}\left(u^{\alpha}\left(\exp \left(H_{i} \Delta \widetilde{W}_{s}^{i}\right)\left(V_{s-}\right)\right)-V_{s-}^{\alpha}-H_{i} u^{\alpha}\left(U_{s-}\right) \Delta \widetilde{W}_{s}^{i}\right) \\
& =V_{0}^{\alpha}+\int_{0}^{t} H_{i}\left(u^{\alpha} \circ R_{g_{s}}\right)\left(U_{s-}\right) \circ \mathrm{d} W_{s}^{i}+\sum_{0<s \leq t}\left(V_{s}^{\alpha}-V_{s-}^{\alpha}-H_{i}\left(u^{\alpha} \circ R_{g_{s}}\right)\left(U_{s-}\right) \Delta W_{s}^{i}\right),
\end{aligned}
$$

where we have used (4.17) and Lemma A.4. We also define $\rho^{\alpha}=u^{\alpha} \circ \rho$ so that we can alternatively write $V_{t}^{\alpha}=\rho^{\alpha}\left(U_{t}, g_{t}\right)$ and we use Itô's formula to compute

$$
\begin{aligned}
V_{t}^{\alpha} & =V_{0}^{\alpha}+\int_{0}^{t} \frac{\partial \rho^{\alpha}}{\partial u^{\beta}}\left(U_{s-}, g_{s}\right) \circ \mathrm{d} U_{s}^{\beta}+\int_{0}^{t} \frac{\partial \phi^{\alpha}}{\partial h^{k}}\left(U_{s-}, g_{s}\right) \circ \mathrm{d} g_{s}^{k} \\
& +\sum_{0<s \leq t}\left(\rho^{\alpha}\left(U_{s}, g_{s}\right)-\rho^{\alpha}\left(U_{s-}, g_{s}\right)-\frac{\partial \rho^{\alpha}}{\partial u^{\beta}}\left(U_{s-}, g_{s}\right) \Delta U_{s}^{\beta}\right) \\
& =V_{0}^{\alpha}+\int_{0}^{t} \frac{\partial \rho^{\alpha}}{\partial u^{\beta}}\left(U_{s-}, g_{s}\right) H_{i} u^{\beta}\left(U_{s-}\right) \circ W_{s}^{i} \\
& +\sum_{0<s \leq t} \frac{\partial \rho^{\alpha}}{\partial u^{\beta}}\left(U_{s-}, g_{s}\right)\left(U_{s}^{\beta}-U_{s-}^{\beta}-H_{i} u^{\beta}\left(U_{s-}\right) \Delta W_{s}^{i}\right)+\int_{0}^{t} \frac{\partial \phi^{\alpha}}{\partial h^{k}}\left(U_{s-}, g_{s}\right) \circ \mathrm{d} g_{s}^{k} \\
& +\sum_{0<s \leq t}\left(V_{s}^{\alpha}-V_{s-}^{\alpha}-\frac{\partial \rho^{\alpha}}{\partial u^{\beta}}\left(U_{s-}, g_{s}\right) \Delta U_{s}^{\beta}\right),
\end{aligned}
$$

where we used that $g_{t}$ is continuous and an analogous version of (4.18) for $U$. Since

$$
\frac{\partial \rho^{\alpha}}{\partial u^{\beta}}\left(U_{s-}, g_{s}\right) H_{i} u^{\beta}\left(U_{s-}\right)=H_{i}\left(u^{\alpha} \circ R_{g_{s}}\right)\left(U_{s-}\right)
$$


holds by the chain rule, we see by comparing the above expressions for $V_{t}^{\alpha}$ that

$$
\int_{0}^{t} \frac{\partial \phi^{\alpha}}{\partial h^{k}}\left(U_{s-}, g_{s}\right) \circ \mathrm{d} g_{s}^{k}=0
$$

holds for all $\alpha$. Since the matrix $\left[\frac{\partial \phi^{\alpha}}{\partial h^{k}}\right]_{k}^{\alpha}$ is of full rank (this is true since $g \mapsto \rho(u, g)$ is an embedding), we can conclude that

$$
\int_{0}^{t} 1 \circ \mathrm{d} g_{s}^{k}=0
$$

holds for all $k$. Therefore, the process $g_{t}$ is constant and hence constantly equal to identity. This proves that the horizontal lift and anti-development are unique and this completes the proof of the theorem.

\subsection{Proof of Theorem 2.14}

We already know from Theorem 2.19 that the anti-development (when it exists) is uniquely determined on $[0, \tau)$ by the pair of processes $X$ and $J$. Due to a particular jump structure of $X$ inferred from its generator, we will see that there will always exist a process $J$ satisfying Definition 2.17, but we need to find an appropriate one such that the anti-development will be a Euclidean Lévy process.

Hence, for the proof of Theorem 2.14 we need to construct a suitable jump process $J$ (on the extended probability space) and show that the associated anti-development is a Lévy process with given characteristics. Since the anti-development is a priori only defined on $[0, \tau)$, we also need to show that it can be extended past $\tau$.

We will first focus on the construction of the appropriate jump process $J$, or more precisely, on the construction of the associated random measure on $\mathbb{R}^{d} \times \mathbb{R}_{+}$. This simple random measure, which we will also denote by $J$ has (random) unit atoms at $\left(s, J_{s}\right)$ when $J_{s} \neq 0$. Before constructing it, we want to characterize it. The following lemma will be useful.

Lemma 4.5. Let $G$ be a subgroup of $\mathrm{GL}(d)$ and let $N$ be a Poisson random measure on $\mathbb{R}_{+} \times \mathbb{R}^{d}$ with a mean measure $\mathcal{L} e b \otimes \nu$, where $\nu$ is a $G$-invariant Lévy measure. Further, let $\left(g_{t}\right)_{t \in \mathbb{R}_{+}}$be a predictable process with values in $G$. Denote by $\Phi$ a random transformation of $\mathbb{R}_{+} \times \mathbb{R}^{d}$ given by

$$
\Phi(\omega, t, z)=\left(t, g_{t}(\omega) z\right)
$$

Then the transformed (push-forward) measure $\Phi_{*} N:=N \Phi^{-1}$ is also a Poisson random measure on $\mathbb{R}_{+} \times \mathbb{R}^{d}$ with a mean measure $\mathcal{L} e b \otimes \nu$.

Proof. The compensator measure of $N$ is its mean measure $\mu:=\mathcal{L} e b \otimes \nu$. It is enough to show that $\Phi_{*} \mu=\mu$, as we can then use [22, Thm. 2.1] to deduce the claim. Let us take an arbitrary $I \in \mathfrak{B}\left(\mathbb{R}_{+}\right), A \in \mathfrak{B}\left(\mathbb{R}^{d}\right)$ and then

$$
\Phi_{*} \mu(\omega)(I \times A)=\mu\left(\Phi^{-1}(\omega)(I \times A)\right)=\int_{I} \nu\left(g_{t}(\omega) A\right) \mathrm{d} t=\int_{I} \nu(A) \mathrm{d} t=\mathcal{L} e b(I) \nu(A)
$$

holds for almost every $\omega$, where we used Fubini-Tonelli theorem in the second equality and a $G$-invariance of the measure $\nu$ in the third equality. Since sets $I \times A$ generate $\mathfrak{B}\left(\mathbb{R}_{+} \times \mathbb{R}^{d}\right)$, we have shown that $\Phi_{*} \mu$ is non-random and equal to $\mu$, so the claim of the lemma follows.

Therefore, if $Y$ is a Lévy process, with jumps given by a Poisson random measure $N$, which is an anti-development of $X$, then we know that $J$ needs to be given as a 
transformation of $N$ where each atom at $(t, z)$ with $t<\tau$ is transformed to an atom at $\left(t, q^{-1}\left(X_{t-}\right) U_{t-} z\right)$, where $U$ is solution of Marcus SDE (2.4) and $q$ is a section of the holonomy bundle as in Section 2.4. We do not transform the atoms at $(t, z)$ with $t \geq \tau$. But $q^{-1}\left(X_{-}\right) U_{-}$is a predictable $\operatorname{Hol}(u)$-valued process (we trivially extend it past $\tau$ ) and Lévy measure $\nu$ of $Y$ is $\operatorname{Hol}(u)$-invariant, hence Lemma 4.5 implies that $J$ is also a Poisson random measure with mean measure $\mathcal{L} e b \otimes \nu$. Note that the condition equivalent to Definition 2.17 which relates the random measure $J$ and a process $X$ is

$$
J(\{(s, z)\})=1 \Longrightarrow X_{s}=\operatorname{Exp}_{X_{s-}}\left(q\left(X_{s-}\right) z\right), J\left(\{s\} \times \mathbb{R}^{d}\right)=0 \Longrightarrow X_{s}=X_{s-} \text { for } s<\tau \text {. }
$$

Hence the goal is to find suitable $J$ which is a Poisson random measure and we have the following result.

Proposition 4.6. Let $X$ be a Markov process on $M$ adapted to $\left(\mathcal{H}_{t}\right)_{t \in \mathbb{R}_{+}}$with the generator $\mathscr{L}_{X}$ given by (2.7). Then on the extended probability space there exists a Poisson random measure $J$ on $\mathbb{R}_{+} \times \mathbb{R}^{d}$ with a mean measure $\mathcal{L} e b \otimes \nu$ and adapted to an extended filtration $\left(\mathcal{G}_{t}\right)_{t \in \mathbb{R}_{+}}$such that (4.19) holds.

Before proceeding with the proof, we discuss the phenomena that may occur when constructing $J$. These phenomena illustrate why typically the process $X$ alone does not possess sufficient information and randomness (even on $[0, \tau)$ ) to carry out the construction.

(i) Let $X_{s}=y \neq X_{s-}=x$, such that $B_{x y}:=\left\{z \in \mathbb{R}^{d} \backslash\{0\} ; \operatorname{Exp}_{x}(q(x) z)=y\right\}$ contains exactly one element $z^{\prime}$. Then it is clear that we need to put $J\left(\left\{\left(s, z^{\prime}\right)\right\}\right)=1$.

(ii) Again let $X_{s}=y \neq X_{s-}=x$, but now the set $B_{x y}$ contains more than one element ${ }^{5}$. Of course, it is clear that we should have $J\left(\{s\} \times B_{x y}\right)=1$, but it is not clear which element should be chosen as the atom. If we simply fixed one possible element of $B_{x y}$ for every pair $(x, y)$, this would correctly prescribe the jumps of $X$ and satisfy (4.19), but $J$ might not have the correct intensity measure $\mathcal{L} e b \otimes \nu$. Therefore, in this case we need some additional randomness to appropriately randomly assign the atom on the set $B_{x y}$.

(iii) Finally, it is possible that $X_{s}=X_{s-}=x$ and there can still be jumps (atoms) in $J$ (see also the discussion following Definition 2.15). This can happen since the set $B_{x}:=\left\{z \in \mathbb{R}^{d} \backslash\{0\} ; \operatorname{Exp}_{x}(q(x) z)=x\right\}$ may be non-empty. Thus, even if $J\left(\{s\} \times B_{x}\right)=1$, actually no jump of $X$ occurs at time $s$. Naively, we might simply ignore these jumps, but then again the intensity measure of $J$ might not be equal to $\mathcal{L} e b \otimes \nu$. Therefore, if we wish to get a correct Poisson random measure, we need to add some of these jumps (atoms) as well, even though they will not manifest themself as jumps of $X$. They will have to be taken from an additional (independent) Poisson random measure on the extended probability space which we will also have to use to define $J$ after time $\tau$.

This illustrates that for a general process $X$ we will need to extend our probability space (and filtration) to allow for this additional randomness. This is done in [8, Section 4 ], albeit they only consider a measure $\nu$ on a real line, but essentially every step generalizes to our case of a multidimensional measure. The only step which needs some adjustment due to a slightly different setup is the following. For ease of notation, put $k(x, z)=\operatorname{Exp}_{x}(q(x) z)$ and note that we could have written the jumps in the integral part of generator $\mathscr{L}_{X}$ in (2.7) via the kernel $K: M \times \mathcal{B}(M) \rightarrow \mathbb{R}_{+}$defined by

$$
K(x, A)=\int_{\mathbb{R}^{d}} \mathbb{1}(k(x, z) \in A) \nu(\mathrm{d} z) \text { for } A \in \mathcal{B}(M \backslash\{x\}),
$$

\footnotetext{
${ }^{5}$ These sets are almost surely non-empty due to the form of a generator of $X$.
} 
and this means that the equivalent of [8, Lemma 3.4] holds. Furthermore, the following equivalent of [8, Lemma 4.32] holds.

Lemma 4.7. Let $K$ be a kernel as above. Then there exists a measurable function $\widetilde{k}: M \times M \times(0,1] \rightarrow \mathbb{R}^{d}$, such that

$$
\int_{0}^{1} \int_{A} \mathbb{1}(\widetilde{k}(x, y, u) \in B) K(x, \mathrm{~d} y) \mathrm{d} u=\int_{B} \mathbb{1}(k(x, z) \in A) \nu(\mathrm{d} z)
$$

for every $A \in \mathcal{B}(M), B \in \mathcal{B}\left(\mathbb{R}^{d}\right)$ and $x \in M$. Moreover, for every $x \in M$ and $K(x, \mathrm{~d} y) \mathrm{d} u$ almost every $(y, u)$ we have

$$
k(x, \widetilde{k}(x, y, u))=y .
$$

Proof of Proposition 4.6. Lemma 4.7 allows us to deduce as in [8, §4c, Eq. (4.41) and Prop. 4.42] that we can construct a random measure $\bar{J}$ on the extended probability space, such that (4.19) holds (with $\bar{J}$ taking the role of $J$ ) and such that the compensator measure of $\bar{J}$ is given by $\mathbb{1}\left(k\left(X_{t-}, z\right) \neq X_{t-}, t<\tau\right) \mathrm{d} t \nu(d z)$. Note that the indicator appears, since only observable jumps of $X$ are used during the construction of the random measure. Measure $\bar{J}$ is almost the required random measure. To add the missing part, consider a further extension of the probability space, which supports an independent Poisson random measure $N$ with an intensity measure $\mathcal{L} e b \otimes \nu$, and define a random measure

$J(\mathrm{~d} t, \mathrm{~d} z)=\bar{J}(\mathrm{~d} t, \mathrm{~d} z)+\left(\mathbb{1}\left(k\left(X_{t-}, z\right)=X_{t-}, \bar{J}\left(\{t\} \times \mathbb{R}^{d}\right)=0, t<\tau\right)+\mathbb{1}(t \geq \tau)\right) N(\mathrm{~d} t, \mathrm{~d} z)$.

It is easy to see that its compensator measure is a non-random measure $\mathcal{L} e b \otimes \nu$, which means that $J$ is a Poisson random measure with mean measure $\mathcal{L} e b \otimes \nu$. Furthermore, since added jumps (atoms) from the measure $N$ do not manifest themself as jumps of $X$, the condition (4.19) still holds and this finishes the proof of proposition.

Proof of Theorem 2.14. Proposition 4.6 allows us to construct a Poisson random measure $J$ and hence the associated jump process $J$ which represents jumps of $X$. Therefore, by Theorem 2.19 there exists a uniquely determined anti-development on $[0, \tau)$ induced by $X$ and $J$ and we denote it by $Y$. The only thing left to do is to show that $Y$ can be extended past $\tau$ so that it is a Lévy process with correct characteristics.

First, note that the jump measure of $Y$ is given as a transformation of $J$ where each atom at $(t, z)$ is transformed to an atom at $\left(t, U_{t-}^{-1} q\left(X_{t-}\right) z\right)$, and again Lemma 4.5 implies that the jump measure of $Y$ is exactly a Poisson random measure with mean measure $\mathcal{L} e b \otimes \nu$ restricted to times smaller than $\tau$.

To determine the remaining characteristics, we recall that $Y$ is a semimartingale on a stochastic interval $[0, \tau)$. We wish to use the notion of semimartingale characteristics which are classically defined for semimartingales with infinite lifetime. However, as the Appendix in [38] shows, essentially every result about semimartingales can be extended to semimartingales defined up to a predictable stopping time.

Recall that every semimartingale $Y$ has a representation $Y=Y_{0}+M+A$, where $M$ is a local martingale started at 0 and $A$ is a process of locally finite variation, but this decomposition is not unique. To get uniqueness we define a process $Y_{t}^{e}=\sum_{s<t} \Delta Y_{s} \mathbb{1}\left(\left|\Delta Y_{s}\right| \geq 1\right)$ and then a process $Y-Y^{e}$ with bounded jumps is a special semimartingale, meaning that it has a unique representation $Y-Y^{e}=M+A$, where $A$ is a predictable finite variation process and $M$ is a local martingale. Then the local characteristics $(A, C, \widetilde{\Gamma})$ of a semimartingale $Y$ as in [21, Chapter II] are given by:

(i) $A=\left(A^{j}\right)_{j=1, \ldots, n}$ is a unique predictable finite variation process in the decomposition of a special semimartingale $Y-Y^{e}$, 
(ii) $C=\left(C^{i j}\right)_{i, j=1, \ldots, n}$ is the quadratic variation of the continuous local martingale part $Y^{c}$ of $Y$ (or equivalently of $M$ ) and is given by $C^{i j}=\left[Y^{i, c}, Y^{j, c}\right]$,

(iii) $\widetilde{\Gamma}$ is a predictable compensator of the jump measure $\Gamma(\mathrm{d} t, \mathrm{~d} y)=\sum_{s \leq t} \mathbb{1}\left(\left|\Delta Y_{s}\right|>\right.$ $0) \delta_{\left(s, \Delta Y_{s}\right)}(\mathrm{d} t, \mathrm{~d} y)$ associated to the process $Y$.

We have already established, that a jump measure of $Y$ is a Poisson random measure, hence the third characteristic is equal to a non-random measure $\mathcal{L} e b \otimes \nu$.

The semimartingale $Y$ can be written explicitly via its characteristics as in Theorem II.2.34 in [21] and plugging this decomposition into the defining property (2.2) of Marcus SDE (2.4) for a function $f \circ \pi$, where $f \in C^{\infty}(M)$, we see that

$$
\begin{aligned}
f\left(X_{t}\right) & -f\left(X_{0}\right)-\int_{0}^{t}\left(H_{i}(f \circ \pi)\left(U_{s-}\right) \mathrm{d} A^{i}+\frac{1}{2} H_{i} H_{j}(f \circ \pi)\left(U_{s-}\right) \mathrm{d} C_{s}^{i j}\right) \\
& -\int_{0}^{t} \int_{\mathbb{R}^{d} \backslash\{0\}}\left(f\left(\operatorname{Exp}_{X_{s-}}\left(U_{s-} x\right)\right)-f\left(X_{s-}\right)-\mathbb{1}(|x|<1) x^{i} H_{i}(f \circ \pi)\left(U_{s-}\right)\right) \widetilde{\Gamma}(\mathrm{d} s, \mathrm{~d} x)
\end{aligned}
$$

is a local martingale. We can compare the above expression to the generator $\mathscr{L}_{X}$ from (2.7) and we get

$$
A_{t}^{j}=b^{j} t, \quad C_{t}^{i j}=a^{i j} t, \quad \widetilde{\Gamma}(\mathrm{d} t, \mathrm{~d} z)=\mathrm{d} t \nu(\mathrm{d} z),
$$

which are the characteristics of $Y$ for $t<\tau$. Note that by observing the generator we can only uniquely determine $A$ and $C$, and there can be more than one measure $\widetilde{\Gamma}$ for which the above equality holds (this can happen since functions $z \mapsto k(x, z)$ from above are not necessarily injective). However, we have already identified the third characteristic without directly referring to the generator.

By [21, Thm. II.4.19] we know that ("constant") characteristics as in (4.20) imply that the semimartingale is actually a Lévy process. However, $Y$ is only defined for times in $[0, \tau)$ and we need to extend it to all times in $\mathbb{R}_{+}$. If we can find a Lévy process $\widetilde{Y}=\left(\widetilde{Y}_{t}\right)_{t \in \mathbb{R}_{+}}$with characteristics (4.20) such that $\widetilde{Y}_{t}=Y_{t}$ for $t \in[0, \tau)$, then $\widetilde{Y}$ is also an anti-development of $X$ and it is exactly the Lévy process we needed to construct to prove the theorem. Existence of such a process $\widetilde{Y}$ is guaranteed by the following lemma and this concludes the proof of the theorem.

Lemma 4.8. Let $Y$ be a semimartingale on $[0, \tau)$ with characteristics (4.20) for $t<\tau$, where $\tau$ is a predictable stopping time. Then there exists a Lévy process $\widetilde{Y}=\left(\widetilde{Y}_{t}\right)_{t \in \mathbb{R}_{+}}$ with characteristics (4.20) such that $\widetilde{Y}_{t}=Y_{t}$ for $t \in[0, \tau)$.

Proof. Let $\left\{\tau_{n} ; n \in \mathbb{N}\right\}$ be an announcing sequence of $\tau$. Consider an additional Lévy process $Z$ with characteristics (4.20) which is independent of $Y$. For every $n \in \mathbb{N}$ we may define a process $Z^{(n)}=\left(Z_{t}^{(n)}\right)_{t \in \mathbb{R}_{+}}$by

$$
Z_{t}^{(n)}:=Y_{t}^{\tau_{n}}+\mathbb{1}\left(t \geq \tau_{n}\right)\left(Z_{t}-Z_{\tau_{n}}\right)
$$

for $t \in \mathbb{R}_{+}$. Since characteristics of $Z^{(n)}$ are also given by (4.20), it is a Lévy process and obviously $Z_{t}^{(n)}=Y_{t}$ holds for $t \in\left[0, \tau_{n}\right]$. Lévy process $Z^{(n)}$ has the Lévy-Itô decomposition

$$
Z_{t}^{(n), i}=b^{i} t+\sigma_{j}^{i} B_{t}^{(n), j}+\int_{0}^{t} \int_{0<|x|<1} x^{i} \widetilde{N^{(n)}}(\mathrm{d} s, \mathrm{~d} x)+\int_{0}^{t} \int_{|x| \geq 1} x^{i} N^{(n)}(\mathrm{d} s, \mathrm{~d} x)
$$

for $i=1, \ldots, d$, where $B^{(n)}$ is a standard Brownian motion and $N^{(n)}$ a Poisson random measure with mean measure $\mathcal{L} e b \otimes \nu$. Note that $N^{(n)}$ is equal to the jump measure $\Gamma$ of $Y$ on $\left[0, \tau_{n}\right]$. Furthermore, for $m<n$ we deduce from $Z_{t}^{(n)}=Z_{t}^{(m)}$ for $t \in\left[0, \tau_{m}\right]$ that also 
$B_{t}^{(n)}=B_{t}^{(m)}$ for $t \in\left[0, \tau_{m}\right]$, so that we may unambiguously define a continuous process $B=\left(B_{t}\right)_{t \in[0, \tau)}$ by $B_{t}=B_{t}^{(n)}$ for $t \in\left[0, \tau_{n}\right]$.

Let us assume that there exists a Brownian motion $\widetilde{B}=\left(\widetilde{B}_{t}\right)_{t \in \mathbb{R}_{+}}$such that $\widetilde{B}_{t}=B_{t}$ for $t \in[0, \tau)$. We may then consider a Poisson random measure $N^{\prime}$ with mean measure $\mathcal{L} e b \otimes \nu$ which is independent of $Y$ and $\widetilde{B}$ and define a random measure

$$
N(\mathrm{~d} t, \mathrm{~d} x):=\mathbb{1}(t<\tau) \Gamma(\mathrm{d} t, \mathrm{~d} x)+\mathbb{1}(t \geq \tau) N^{\prime}(\mathrm{d} t, \mathrm{~d} x),
$$

which is also a Poisson random measure with mean measure $\mathcal{L} e b \otimes \nu$ since $\mathcal{L} e b \otimes \nu$ is its compensator measure. Hence we may define a process $\widetilde{Y}=\left(\widetilde{Y}_{t}\right)_{t \in \mathbb{R}_{+}}$by

$$
\widetilde{Y}_{t}^{i}:=b^{i} t+\sigma_{j}^{i} \widetilde{B}_{t}^{j}+\int_{0}^{t} \int_{0<|x|<1} x^{i} \widetilde{N}(\mathrm{~d} s, \mathrm{~d} x)+\int_{0}^{t} \int_{|x| \geq 1} x^{i} N(\mathrm{~d} s, \mathrm{~d} x)
$$

for $i=1, \ldots, d$. It is clear that $\widetilde{Y}$ is a Lévy process with characteristics (4.20) and since $\widetilde{B_{t}}=B_{t}$ for $t \in[0, \tau)$ and $N=\Gamma$ on $[0, \tau)$ we also have $\widetilde{Y}_{t}=Y_{t}$ for $t \in[0, \tau)$, so $\widetilde{Y}$ is the required process.

Therefore, in order to finish the proof we only have to show the existence of Brownian motion $\widetilde{B}$ as above. Note that since $B_{t}=B_{t}^{(n)}$ for $t \in\left[0, \tau_{n}\right]$, the process $B$ is a continuous local martingale on $[0, \tau)$ (see the definition in [16, Section 3] or in [34, Exercise (1.48) in Ch. IV]) and has a quadratic covariation $\left\langle B^{i}, B^{j}\right\rangle_{t}=\delta^{i j} t$ on $[0, \tau)$ for $i, j=1, \ldots, d$. For $m \in \mathbb{N}$ consider a constant stopping time $T_{m} \equiv m$ and a stopped process $B^{T_{m}}$, which is also a continuous local martingale on $[0, \tau)$. We have a trivial bound $\left\langle B^{T_{m}, i}\right\rangle_{\tau} \leq m$ for $i=1, \ldots, d$, so we can conclude from the Burkholder-DavisGundy inequality [34, Thm. (4.1) in Ch. IV] that $\mathbb{E}\left[\sup _{t<\tau}\left|B_{t}^{T_{m}}\right|\right]<\infty$ and it then follows that the $\operatorname{limit}_{\lim } \rightarrow \tau B_{t}^{T_{m}}$ exists (see the discussion after [16, Def. 3.1] and [34, Exercise (1.48) in Ch. IV]). On $\{\tau<\infty\}$ we have that $T_{m} \geq \tau$ from some sufficiently large $m$ onward, so also the limit $\lim _{t \rightarrow \tau} B_{t}$ exists on the same event. As we may define $B_{\tau}:=\lim _{t \rightarrow \tau} B_{t}$ on $\{\tau<\infty\}$ it becomes meaningful to consider a continuous stopped process $B^{\tau}$ and we can define a continuous process $\left(\widetilde{B}_{t}\right)_{t \in \mathbb{R}_{+}}$by

$$
\widetilde{B}_{t}=B_{t}^{\tau}+\mathbb{1}(t \geq \tau)\left(B_{t}^{\prime}-B_{\tau}^{\prime}\right)
$$

where $\left(B_{t}^{\prime}\right)_{t \in \mathbb{R}_{+}}$is another Brownian motion independent of $B$. It is easily shown by Lévy's characterization that $\widetilde{B}$ is also a Brownian motion and obviously $\widetilde{B}_{t}=B_{t}$ holds for $t \in[0, \tau)$, so it is the required process needed to finish the proof of the lemma.

\subsection{Proofs of results in Section 3.2}

Proof of Proposition 3.1. We recall a Lévy-Itô decomposition of a Lévy process $Y$ as in (4.9). Since $X$ solves Marcus SDE (3.2) we can take $f \in C^{\infty}(G)$ and substitute (4.9) into the defining property of the Marcus SDE to get

$$
\begin{aligned}
f\left(X_{t}\right) & =f\left(X_{0}\right)+b^{i} \int_{0}^{t} V_{i}^{L} f\left(X_{s-}\right) \mathrm{d} s+\sigma_{j}^{i} \int_{0}^{t} V_{i}^{L} f\left(X_{s-}\right) \circ \mathrm{d} B_{s}^{j} \\
& +\int_{0}^{t} \int_{0<|x|<1} x^{i} V_{i}^{L} f\left(X_{s-}\right) \widetilde{N}(\mathrm{~d} s, \mathrm{~d} x)+\int_{0}^{t} \int_{|x| \geq 1} x^{i} V_{i}^{L} f\left(X_{s-}\right) N(\mathrm{~d} s, \mathrm{~d} x) \\
& +\int_{0}^{t} \int_{\mathbb{R}^{d} \backslash\{0\}}\left(f\left(X_{s-} \widetilde{\exp }(x)\right)-f\left(X_{s-}\right)-x^{i} V_{i}^{L} f\left(X_{s-}\right)\right) N(\mathrm{~d} s, \mathrm{~d} x) .
\end{aligned}
$$


After splitting the domain in the last integral and some algebraic manipulation using $\widetilde{N}(\mathrm{~d} s, \mathrm{~d} x)=N(\mathrm{~d} s, \mathrm{~d} x)-\mathrm{d} s \nu(\mathrm{d} x)$ we get

$$
\begin{aligned}
f\left(X_{t}\right) & =f\left(X_{0}\right)+b^{i} \int_{0}^{t} V_{i}^{L} f\left(X_{s-}\right) \mathrm{d} s+\sigma_{j}^{i} \int_{0}^{t} V_{i}^{L} f\left(X_{s-}\right) \circ \mathrm{d} B_{s}^{j} \\
& +\int_{0}^{t} \int_{0<|x|<1}\left(f\left(X_{s-} \widetilde{\exp }(x)\right)-f\left(X_{s-}\right)\right) \widetilde{N}(\mathrm{~d} s, \mathrm{~d} x) \\
& +\int_{0}^{t} \int_{|x| \geq 1}\left(f\left(X_{s-} \widetilde{\exp }(x)\right)-f\left(X_{s-}\right)\right) N(\mathrm{~d} s, \mathrm{~d} x) \\
& +\int_{0}^{t} \int_{0<|x|<1}\left(f\left(X_{s-} \widetilde{\exp }(x)\right)-f\left(X_{s-}\right)-x^{i} V_{i}^{L} f\left(X_{s-}\right)\right) \mathrm{d} s \nu(\mathrm{d} x) .
\end{aligned}
$$

We recall that $\widetilde{\exp }$ is a diffeomorphism from some neighbourhood $O$ of $0 \in \mathbb{R}^{d}$ to some neighbourhood $U$ of the unit $e \in G$ and we may assume that $O \subseteq\left\{x \in \mathbb{R}^{d} ;|x|<1\right\}$. We take a smaller open set $\widetilde{O}$ with $0 \in O$ and $\operatorname{cl}(\widetilde{O}) \subseteq O$ and let $\widetilde{U}:=\widetilde{\exp }(\widetilde{O})$. Then we can find $l \in C^{\infty}\left(\mathbb{R}^{d}, \mathbb{R}^{d}\right)$ such that $l(x)=x$ on $\operatorname{cl}(\widetilde{O})$ and $\operatorname{supp} l \subseteq O$. Given such a function $l$, we define $\xi \in C^{\infty}\left(G, \mathbb{R}^{d}\right)$ by

$$
\xi(h)= \begin{cases}0, & h \in \widetilde{\exp }(\operatorname{supp} l)^{c} \\ l\left(\widetilde{\exp }^{-1}(h)\right), & h \in U .\end{cases}
$$

Function $\xi$ is smooth since it is smooth on both open domains and agrees on their intersection. We can check that $l(x)=\xi(\widetilde{\exp }(x))$ holds. Moreover, simple calculations show that $\xi(e)=0$ and $V_{j}^{L} \xi^{i}(e)=\delta_{j}^{i}$. This means that functions $\xi^{i}$ are suitable coordinate functions on the neighbourhood of $e$ and since $\int_{G} \sum_{i=1}^{d}\left(\xi^{i}(h)\right)^{2} \mu(\mathrm{d} h)=\int_{\mathbb{R}^{d}} \sum_{i=1}^{d}\left(l^{i}(x)\right)^{2} \nu(\mathrm{d} x)<\infty$, $\mu(\{e\})=0$ and $\mu\left(A^{c}\right)=\nu\left(\widetilde{\exp }^{-1}\left(A^{c}\right)\right)<\infty$ for any open $A \ni e$, the measure $\mu$ is a indeed Lévy measure on $G$ (see [28, Ch. 1] for definitions).

Then the last integral in (4.21) can be expressed as

$$
\begin{aligned}
& \int_{0}^{t} \int_{0<|x|<1}\left(f\left(X_{s-} \widetilde{\exp }(x)\right)-f\left(X_{s-}\right)-\xi^{i}(\widetilde{\exp }(x)) V_{i}^{L} f\left(X_{s-}\right)\right) \mathrm{d} s \nu(\mathrm{d} x) \\
& +c^{i} \int_{0}^{t} V_{i}^{L} f\left(X_{s-}\right) \mathrm{d} s
\end{aligned}
$$

where $c^{i}=\int_{0<|x|<1}\left(l^{i}(x)-x^{i}\right) \nu(\mathrm{d} x)$ for $i=1, \ldots, d$ are finite since $l(x)=x$ near 0 . We then plug (4.22) back into (4.21), use the fact that $l(x)=\xi(\widetilde{\exp }(x))=0$ for $|x|>1$ and manipulate the integral in the third line with $\widetilde{N}(\mathrm{~d} s, \mathrm{~d} x)=N(\mathrm{~d} s, \mathrm{~d} x)-\mathrm{d} s \nu(\mathrm{d} x)$ to get

$$
\begin{aligned}
f\left(X_{t}\right) & =f\left(X_{0}\right)+\widetilde{b}^{i} \int_{0}^{t} V_{i}^{L} f\left(X_{s-}\right) \mathrm{d} s+\sigma_{j}^{i} \int_{0}^{t} V_{i}^{L} f\left(X_{s-}\right) \circ \mathrm{d} B_{s}^{j} \\
& +\int_{0}^{t} \int_{\mathbb{R}^{d} \backslash\{0\}}\left(f\left(X_{s-} \widetilde{\exp }(x)\right)-f\left(X_{s-}\right)\right) \widetilde{N}(\mathrm{~d} s, \mathrm{~d} x) \\
& +\int_{0}^{t} \int_{\mathbb{R}^{d} \backslash\{0\}}\left(f\left(X_{s-} \widetilde{\exp }(x)\right)-f\left(X_{s-}\right)-\xi^{i}(\widetilde{\exp }(x)) V_{i}^{L} f\left(X_{s-}\right)\right) \mathrm{d} s \nu(\mathrm{d} x)
\end{aligned}
$$

where $\widetilde{b}^{i}=b^{i}+c^{i}$. Additionally, we define a Poisson random measure $N^{\prime}$ on $\mathbb{R}_{+} \times$ $(G \backslash\{e\})$ by $N^{\prime}\left([0, t], A^{\prime}\right)=N\left([0, t], \widehat{\exp }^{-1}\left(A^{\prime}\right)\right)$, where $A^{\prime} \subseteq G \backslash\{e\}$ is any measurable set. This means that mean measure of $N^{\prime}$ is $\mathcal{L} e b \otimes \mu$ and we also define a compensator $\widetilde{N}^{\prime}\left([0, t], A^{\prime}\right)=N^{\prime}\left([0, t], A^{\prime}\right)-t \mu(A)$. We then use a change-of-variables formula for the 
last two integrals in (4.23) to get

$$
\begin{aligned}
f\left(X_{t}\right) & =f\left(X_{0}\right)+\widetilde{b}^{i} \int_{0}^{t} V_{i}^{L} f\left(X_{s-}\right) \mathrm{d} s+\sigma_{j}^{i} \int_{0}^{t} V_{i}^{L} f\left(X_{s-}\right) \circ \mathrm{d} B_{s}^{j} \\
& +\int_{0}^{t} \int_{G \backslash\{e\}}\left(f\left(X_{s-} h\right)-f\left(X_{s-}\right)\right) \widetilde{N}^{\prime}(\mathrm{d} s, \mathrm{~d} h) \\
& +\int_{0}^{t} \int_{G \backslash\{e\}}\left(f\left(X_{s-} h\right)-f\left(X_{s-}\right)-\xi^{i}(h) V_{i}^{L} f\left(X_{s-}\right)\right) \mathrm{d} s \mu(\mathrm{d} h) .
\end{aligned}
$$

Technically, after using a change-of-variables formula we get integration against measure $\widetilde{\mu}:=\widetilde{\exp }_{*} \nu$ and the Poisson random measure corresponding to that measure with the domain of the integration being the whole group $G$, but since both integrands vanish at $e$, we can instead use $\mu$ and $\widetilde{N}^{\prime}$, and the domain of integration reduces to $G \backslash\{e\}$. This last stochastic integral equation for $X$ is also known as a Lévy-Itô decomposition of a Lévy process in a Lie group (see [28, Thm. 1.2]) and it indeed follows that $X$ is a (left) Lévy process with a Lévy measure $\mu$.

Proof of Proposition 3.2. The only if part is a consequence of Proposition 3.1 so we now focus on the if part. The generator of a (left) Lévy process is identified in [28, Thm. 1.1]. If we are able to find a Lévy measure $\nu$ on $\mathbb{R}^{d}$ such that $\mu=\left.\widetilde{\exp }_{*} \nu\right|_{G \backslash\{e\}}$ holds, we may then reverse the computations in the proof of Proposition 3.1 to express the generator of a left Lévy process in a form on which we may use the characterization from Theorem 2.14 and thus find a required process $Y$.

To construct such a suitable measure $\nu$ we first take open sets $U \subseteq G$ and $O \subseteq \mathbb{R}^{d}$ such that $\widetilde{\exp }: O \rightarrow U$ is a diffeomorphism. This allows us to define a measure $\rho_{O}$ on $O$ by $\rho_{O}=\exp _{*}^{-1}\left(\left.\mu\right|_{U}\right)$. Since $\mu\left(\operatorname{Im}(\exp )^{c}\right)=0$, we can treat $\mu$ as a measure on $\operatorname{Im}(\exp )$. We denote $F=\widehat{\exp }^{-1}\left(U^{c}\right)$ and then the restricted map exp: $F \rightarrow \operatorname{Im}(\exp ) \cap U^{c}$ is still surjective. Since $\mu$ is a finite measure on $\operatorname{Im}(\exp ) \cap U^{c}$ we can then use [12, Prop. 1.101] to show that there exists a measure $\rho_{F}$ on $F$ such that $\widetilde{\exp }_{*} \rho_{F}=\left.\mu\right|_{\operatorname{Im}(\exp ) \cap U^{c}}$. Actually, [12] deals with sub-probability measures but all of the results extend to finite measures. Finally, the measure $\nu$ on $\mathbb{R}^{d}$ is defined by $\nu(A)=\rho_{O}(A \cap O)+\rho_{F}(A \cap F)$ for a measurable set $A$. Clearly $\mu=\left.\widetilde{\exp }_{*} \nu\right|_{G \backslash\{e\}}$ holds and, moreover, $\nu$ is a Lévy measure since $\nu(\{0\})=$ $\mu(\{e\})=0, \nu\left(O^{c}\right)=\mu\left(U^{c}\right)<\infty$ and $\int_{O} \sum_{i=1}^{d}\left(x^{i}\right)^{2} \nu(\mathrm{d} x)=\int_{U} \sum_{i=1}^{d}\left(\xi^{i}(h)\right)^{2} \mu(\mathrm{d} h)<\infty$, where $\xi^{i}$ are coordinate functions introduced in the proof of Proposition 3.1.

\section{A Connections, geodesics, horizontal lift and anti-development}

A (linear) connection is given in terms of a covariant derivative, which prescribes how to derive vector fields along each other. A covariant derivative is a bilinear map $\nabla: \Gamma(T M) \times \Gamma(T M) \rightarrow \Gamma(T M)$, which is additionally $C^{\infty}(M)$-linear in the first argument and obeys a product rule in the second argument i.e. $\nabla_{X}(f Y)=f \nabla_{X} Y+(X f) Y$. Covariant derivatives act locally; to compute $\left(\nabla_{X} Y\right)_{p}$ it is enough to only know the value $X_{p}$ and the values of $Y$ along a curve through $p$ which has a tangent vector $X_{p}$ at the point $p$. In local coordinates $x^{i}$, the vector fields $\partial_{i}:=\frac{\partial}{\partial x^{i}}$ form a local frame, i.e. the tangent vectors $\left.\partial_{i}\right|_{p}$ form a basis of the tangent space $T_{p} M$ at any point $p$ in the local chart. Hence, we can locally express $\nabla_{\partial_{i}} \partial_{j}=\Gamma_{i j}^{k} \partial_{k}$ for some smooth functions $\Gamma_{i j}^{k}$, which are known as Christoffel symbols. Christoffel symbols determine the covariant derivative.

A vector field $\mathcal{X}$ along a curve $\gamma$, is said to be parallel (along the curve $\gamma$ ) if $\nabla_{\dot{\gamma}} \mathcal{X}=0$ holds at every point of the curve. In a local chart a vector field along $\gamma$ can be expressed as $\mathcal{X}_{\gamma_{t}}=\mathcal{X}^{k}(t) \partial_{k}$ and a parallel vector field is then a solution of a particular system of 
Lévy processes on smooth manifolds with a connection

ordinary differential equations (ODEs), namely

$$
\dot{\mathcal{X}}^{k}(t)+\Gamma_{i j}^{k}\left(\gamma_{t}\right) \dot{\gamma}_{t}^{i} \mathcal{X}^{j}(t)=0 .
$$

Once a curve $\gamma$ with $\gamma_{0}=p$ and $v \in T_{p} M$ are fixed, the local existence and uniqueness theorem for ODEs guarantees that there is a unique parallel vector field $\mathcal{X}$ along $\gamma$ with $\mathcal{X}_{p}=v$. Since the system of ODEs is (non-autonomous) linear, the solution cannot explode and is defined along the whole curve $\gamma$.

Actually, we have constructed a unique (parallel) lift of a curve onto a tangent bundle (once the initial point is fixed). Additionally, using parallel vector fields along the curve $\gamma$ with $\gamma_{0}=p$ and $\gamma_{1}=q$ we can connect the tangent spaces $T_{p} M$ and $T_{q} M$. More precisely, for each $v \in T_{p} M$ we define $\tau_{\gamma}(v) \in T_{q} M$ as a value at $q$ of the unique parallel vector field $\mathcal{X}$ (along $\gamma$ ) with $\mathcal{X}_{p}=v$. The map $\tau_{\gamma}$ is called the parallel transport along $\gamma$ and can be shown to be a linear isomorphism.

Covariant derivative also allows us to define geodesics, which are special curves $\gamma$ such that their velocity vector field $\dot{\gamma}$ is parallel along $\gamma$. A geodesic is locally given as a solution of the system of second order ODEs

$$
\ddot{\gamma}_{t}^{k}+\Gamma_{i j}^{k}\left(\gamma_{t}\right) \dot{\gamma}_{t}^{i} \dot{\gamma}_{t}^{j}=0
$$

for $k=1, \ldots, d$ and therefore a geodesic is unique once we fix an initial point and an initial velocity. This allows us to define an exponential map in essentially the same manner as on Riemannian manifolds. A geodesic exponential map is a map $\operatorname{Exp}_{p}: O \subseteq$ $T_{p} M \rightarrow M, \operatorname{Exp}_{p}(X):=\gamma(1)$, where $\gamma$ is a unique geodesic with $\gamma(0)=p$ and $\dot{\gamma}(0)=X$ and $O$ is an open star-shaped neighbourhood of $0 \in T_{p} M$ where the geodesic exponential map is well defined ${ }^{6}$. If the domain of the geodesic exponential map at $p$ is equal to $T_{p}$ for any $p \in M$ we say that $M$ is geodesically complete. Note that by Hopf-Rinow theorem compact Riemannian manifolds are always geodesically complete, but there exist nonmetric connections on compact manifolds which are not geodesically complete [31, Ex. 7.16].

In Section 2.2 we introduced a frame bundle. On the frame bundle a notion of vertical vectors is naturally defined. More precisely, the vertical space is defined by $V_{u} F(M):=\operatorname{ker} d \pi_{u}$. We now show how the covariant derivative can be used to define horizontal vectors and get a splitting required for the connection. Let $u_{t}$ be a smooth curve in $F(M)$ so that $\pi\left(u_{t}\right)$ is a smooth curve in $M$. A curve $u_{t}$ is called horizontal if a vector field $u_{t} e$ is parallel along the curve $\pi\left(u_{t}\right)$ for each $e \in \mathbb{R}^{d}$. Additionally, a vector in $T_{u} F(M)$ is called horizontal if it is a velocity vector of a horizontal curve through $u$. The space of horizontal vectors at $u$ is denoted by $H_{u} F(M)$. It can be easily checked that vertical and horizontal space intersect trivially and furthermore the equality $T_{u} F(M)=V_{u} F(M) \oplus H_{u} F(M)$ holds, giving us the required splitting of tangent spaces of the frame bundle $e^{7}$. It is also easily seen that the following equivariance property holds: $d\left(R_{g}\right)_{u}\left(H_{u} F(M)\right)=H_{u g} F(M)$ for all $g \in \mathrm{GL}(d)$, where $d\left(R_{g}\right)_{u}: T_{u} F(M) \rightarrow T_{u g} F(M)$ is a differential at $u$ of a (right) group action map $R_{g}: u \mapsto u g$.

Since we have the splitting, dimensionality arguments show that the differential $d \pi_{u}$ restricted to the horizontal space is an isomorphism between $H_{u} F(M)$ and $T_{\pi(u)} M$. The inverse of this (restricted) differential defines a horizontal lift of tangent vectors in $M$.

\footnotetext{
${ }^{6}$ The differential equation for a geodesic has a unique solution defined on a maximal time interval. However, this interval might not be all of $\mathbb{R}$. Hence geodesics might explode in finite time and the domain of the geodesic exponential map might have to be restricted.

${ }^{7}$ This kind of splitting is also known as a principal Ehrenmanns connection on a principal fibre bundle, whereas covariant derivative is also known as a linear connection and can be thought as a structure on the tangent bundle $T M$. Since $T M$ is an associated bundle of $F(M)$, (principal) connections on $F(M)$ are naturally induced by linear connections on $T M$ and vice versa [23, Thm. 7.5 in Ch. III].
} 
Moreover, horizontal lifts can be obtained for (piecewise) smooth curves. Let $\gamma$ be a smooth curve in $M$. We call a curve $u_{t}$ in $F(M)$ a horizontal lift of curve $\gamma$ if $\pi\left(u_{t}\right)=\gamma_{t}$ and it is horizontal, i.e. $\dot{u}_{s} \in H_{u_{s}} F(M)$ for all $s$. Once an initial frame $u_{0} \in \pi^{-1}\left(\left\{\gamma_{0}\right\}\right)$ is fixed, the horizontal lift is unique and exists for all times for which the underlying curve $\gamma$ is defined. This can be seen by the following computation in a local chart. A local chart for $O \subseteq M$ around $p$ induces a local chart for $\pi^{-1}(O) \subseteq F(M)$ around $\pi^{-1}(p)$. A frame $u$ above $p$ can be uniquely represented as $u=\left(x^{i}, r_{m}^{k}\right)$ where $u e_{m}=r_{m}^{k} \partial_{k}$. Then the horizontal lift of $\gamma$ is given as $\left(\gamma_{t}^{i}, r_{m}^{k}(t)\right)$, where $r_{m}^{k}$ satisfy a system of linear ODEs

$$
\dot{r}_{m}^{k}(t)+r_{m}^{l}(t) \Gamma_{j l}^{k}\left(\gamma_{t}\right) \dot{\gamma}_{t}^{j}=0 .
$$

Uniqueness and existence of the horizontal lift after fixing the initial frame thus follows from the theory of ODEs and linearity of the system implies non-explosion.

Furthermore, if $u_{t}$ is a horizontal lift of $\gamma$ with an initial frame $u_{0}$, then $u_{t} g$ is a horizontal lift of $\gamma$ with an initial frame $u_{0} g$ for any $g \in \mathrm{GL}(d)$. In particular, we recover parallel transport as $\tau_{\gamma}=u_{1} u_{0}^{-1}$ for any horizontal lift $u_{t}$ of $\gamma$, since the quantity on the right hand side does not depend on the initial frame (and hence the particular horizontal lift).

Horizontal spaces can also be described by a family of horizontal vector fields. For any $e \in \mathbb{R}^{d}$ a fundamental horizontal vector field $H_{e}$ is defined by $H_{e}(u):=\left(d \pi_{u}\right)^{-1}(u e) \in$ $H_{u} F(M)$ for any frame $u$. Since the definition is pointwise, the smoothness is not immediate, but quickly follows from the following representation in local coordinates taken from [18, Prop. 2.1.3].

Lemma A.1. In terms of a local chart on $F(M)$ where $u=\left(x^{i}, r_{m}^{k}\right)$, we have

$$
H_{e}(u)=e^{i} r_{i}^{j} \frac{\partial}{\partial x^{j}}-e^{i} r_{i}^{j} r_{m}^{l} \Gamma_{j l}^{k}(x) \frac{\partial}{\partial r_{m}^{k}},
$$

where $u=(x, r)=\left(x^{i}, r_{m}^{k}\right) \in F(M)$ and $e=\left(e^{i}\right)$.

It is trivially seen that the map $e \mapsto H_{e}$ is linear. Let $e_{1}, \ldots, e_{d}$ denote the standard base of $\mathbb{R}^{d}$, then $H_{i}:=H_{e_{i}}$ and vector fields $H_{1}, \ldots, H_{d}$ span the whole horizontal space $H_{u} F(M)$ at any frame $u$. The following lemma, taken from [23, Prop. III.2.2], relates the fundamental horizontal fields at different frames above the same point in the manifold.

Lemma A.2. Let $e \in \mathbb{R}^{d}$ and $g \in \mathrm{GL}(d)$. Then

$$
H_{e}(u g)=d\left(R_{g}\right)_{u}\left(H_{g e}(u)\right)
$$

for every $u \in F(M)$, where $d\left(R_{g}\right)_{u}: T_{u} F(M) \rightarrow T_{u g} F(M)$ is a differential at $u$ of a (right) group action map $R_{g}: u \mapsto u g$.

The following two lemmas are easy corollaries.

Lemma A.3. For any $u \in F(M), e \in \mathbb{R}^{d}, g \in \mathrm{GL}(d)$ we have $\exp \left(H_{e}\right)(u g)=\exp \left(H_{g e}\right)(u)$. $g$.

Proof. If $\psi$ is an integral curve of $H_{g e}$ with $\psi(0)=u$, then Lemma A.2 implies that $\widetilde{\psi}:=\psi g$ is an integral curve of $H_{e}$ with $\widetilde{\psi}(0)=u g$ and the claim follows from the uniqueness of integral curves and the definition of the flow exponential map.

Lemma A.4. For every smooth function $f \in C^{\infty}(F(M)), x_{1}, \ldots, x_{m} \in \mathbb{R}^{d}$ and $g \in \mathrm{GL}(d)$ we have

$$
\left(H_{x_{1}} \cdots H_{x_{m}} f\right) \circ R_{g}=H_{g x_{1}} \cdots H_{g x_{m}}\left(f \circ R_{g}\right) .
$$


Proof. We prove the lemma by induction on $m$. The base case of $m=1$ is just a restatement of Lemma A.2. For an inductive step let the claim hold for $m$ vectors in $\mathbb{R}^{d}$ and take an additional vector $x_{m+1}$. Denote $g=H_{x_{m+1}} f$ and then

$$
\begin{aligned}
\left(H_{x_{1}} \cdots H_{x_{m+1}} f\right) \circ R_{g} & =\left(H_{x_{1}} \cdots H_{x_{m}} g\right) \circ R_{g} \\
& =H_{g x_{1}} \cdots H_{g x_{m}}\left(g \circ R_{g}\right) \\
& =H_{g x_{1}} \cdots H_{g x_{m+1}}\left(f \circ R_{g}\right),
\end{aligned}
$$

where the second equality holds by the induction hypothesis and the last equality by the base case $m=1$.

So far we were able to start with a curve $\gamma$ in $M$ and after fixing an initial frame $u_{0}$ we could construct a unique horizontal lift $u_{t}$ in the frame bundle $F(M)$. We can go one step further and define an anti-development of a curve $\gamma$ (or of its lift $u_{t}$ ) by

$$
w_{t}:=\int_{0}^{t} u_{s}^{-1}\left(\dot{\gamma}_{s}\right) \mathrm{d} s,
$$

which is a smooth curve in $\mathbb{R}^{d}$ started at 0 . One could also reverse the construction and start with a curve $w_{t}$ in $\mathbb{R}^{d}$ and construct a horizontal curve $u_{t}$ on $F(M)$ (and a curve $\gamma_{t}=\pi\left(u_{t}\right)$ on $M$ ) whose anti-development is $w_{t}$. Once we fix the initial frame this correspondence is unique and $u_{t}$ is given as a solution of the ODE

$$
\dot{u}_{t}=H_{i}\left(u_{t}\right) \dot{w}_{t}^{i} .
$$

Therefore, we obtain a one-to-one correspondence between a (piecewise) smooth curves in $M$, their horizontal lifts in $F(M)$ and their anti-developments in $\mathbb{R}^{d}$ once the initial frame is fixed.

It is interesting to check how this correspondence is expressed for special curves geodesics. One quickly finds that the anti-development of a geodesic is a uniform motion, i.e. a constant speed straight line. This simplifies the ODE that the horizontal lift $u_{t}$ is a solution of and actually shows that $u_{t}$ is an integral curve of some fundamental horizontal vector field, i.e. there exists some $e \in \mathbb{R}^{d}$ such that $\dot{u}_{t}=H_{e}\left(u_{t}\right)$. Therefore, uniform motion in $\mathbb{R}^{d}$ corresponds to moving along an integral curve of some fundamental horizontal vector field or equivalently moving along a geodesic in $M$. This also relates flow exponential map ${ }^{8}$ on $F(M)$ to a geodesic exponential map on $M$ so that the equality

$$
\pi\left(\exp \left(H_{e}\right)(u)\right)=\operatorname{Exp}_{p}\left(d \pi_{u}\left(H_{e}\right)\right)=\operatorname{Exp}_{p}(u e)
$$

holds for any $e \in \mathbb{R}^{d}$ and any frame $u$ with $\pi(u)=p$.

\section{References}

[1] D. Applebaum, A horizontal Lévy process on the bundle of orthonormal frames over a complete Riemannian manifold, Séminaire de probabilités de Strasbourg 29 (1995), 166-180 (en). MR1459458

[2] D. Applebaum, Lévy Processes and Stochastic Calculus, Cambridge Studies in Advanced Mathematics, Cambridge University Press, 2004. MR2072890

[3] D. Applebaum and A. Estrade, Isotropic Lévy processes on Riemannian manifolds, The Annals of Probability 28 (2000), no. 1, 166-184. MR1756002

[4] D. Applebaum and H. Kunita, Lévy flows on manifolds and Lévy processes on Lie groups, J. Math. Kyoto Univ. 33 (1993), no. 4, 1103-1123. MR1251218

\footnotetext{
${ }^{8}$ We have only defined a flow of complete fields, but analogously we could define a local flow of a (noncomplete) field.
} 
Lévy processes on smooth manifolds with a connection

[5] D. Applebaum and M. Liao, Markov Processes with Jumps on Manifolds and Lie Groups, Geometry and Invariance in Stochastic Dynamics, to appear.

[6] D. Applebaum and R. Shewell Brockway, $L^{2}$ properties of Lévy Generators on Compact Riemannian Manifolds, Journal of Theoretical Probability (2020). MR4259458

[7] J. Bertoin, Lévy Processes, Cambridge Tracts in Mathematics 121, Cambridge University Press, 1996. MR1406564

[8] E. Çinlar and J. Jacod, Representation of Semimartingale Markov Processes in Terms of Wiener Processes and Poisson Random Measures, Seminar on Stochastic Processes, 1981 (E. Çinlar, K. L. Chung, and R. K. Getoor, eds.), Birkhäuser Boston, 1981, pp. 159-242. MR0647786

[9] E. Çinlar, J. Jacod, Philip Protter, and M. Sharpe, Semimartingales and Markov processes, Probability Theory and Related Fields 54 (1980), 161-219. MR0597337

[10] S. Cohen, Géométrie différentielle stochastique avec sauts 1, Stochastics and Stochastic Reports 56 (1996), no. 3-4, 179-203. MR1396760

[11] S. Cohen, Géométrie différentielle stochastique avec sauts 2: discrétisation et applications des eds avec sauts, Stochastics and Stochastic Reports 56 (1996), no. 3-4, 205-225. MR1396761

[12] E.E. Doberkat, Stochastic Relations: Foundations for Markov Transition Systems, Chapman \& Hall/CRC Studies in Informatics Series, CRC Press, 2007. MR2345820

[13] E. B. Dynkin, Markov processes: Volume 1, 1 ed., Die Grundlehren der Mathematischen Wissenschaften 121/122, Springer-Verlag Berlin Heidelberg, 1965. MR0193671

[14] A. Estrade, A characterization of Markov solutions for stochastic differential equations with jumps, Séminaire de probabilités de Strasbourg 31 (1997), 315-321 (en). MR1478740

[15] T. Fujiwara, Stochastic differential equations of jump type on manifolds and Lévy flows, J. Math. Kyoto Univ. 31 (1991), no. 1, 99-119. MR1093330

[16] R. K. Getoor and M. J. Sharpe, Conformal martingales, Inventiones Mathematicae 16 (1972), no. 4, 271-308. MR0305473

[17] J. Hano and H. Ozeki, On the holonomy groups of linear connections, Nagoya Math. J. 10 (1956), 97-100. MR0082164

[18] E.P. Hsu, Stochastic Analysis on Manifolds, Graduate Studies in Mathematics 38, vol. 38, American Mathematical Society, 2002. MR1882015

[19] G. A. Hunt, Semi-Groups of Measures on Lie Groups, Transactions of the American Mathematical Society 81 (1956), no. 2, 264-293. MR0079232

[20] J. Jacod and P. Protter, Une remarque sur les equations differentielles stochastiques a solutions markoviennes, Séminaire de Probabilités XXV (Berlin, Heidelberg) (Jaques Azéma, Marc Yor, and Paul André Meyer, eds.), Springer Berlin Heidelberg, 1991, pp. 138-139. MR1187777

[21] J. Jacod and A. N. Shiryaev, Limit Theorems for Stochastic Processes, 2 ed., Grundlehren der mathematischen Wissenschaften 288, Springer-Verlag Berlin Heidelberg, 2003. MR1943877

[22] O. Kallenberg, Random time change and an integral representation for marked stopping times, Probability Theory and Related Fields 86 (1990), no. 2, 167-202. MR1065278

[23] S. Kobayashi and K. Nomizu, Foundations of Differential Geometry, Wiley Classics Library, vol. 1, Wiley-Interscience, 1963. MR0152974

[24] H. Kunita, Stochastic flows and jump-diffusions, 1 ed., Probability Theory and Stochastic Modelling, Springer, 2019. MR3929750

[25] T. G. Kurtz, É. Pardoux, and P. Protter, Stratonovich stochastic differential equations driven by general semimartingales, Annales de l'I.H.P. Probabilités et Statistiques 31 (1995), no. 2, 351-377 (en). MR1324812

[26] A.E. Kyprianou, Fluctuations of Lévy Processes with Applications: Introductory Lectures, Universitext, Springer Berlin Heidelberg, 2014. MR3155252

[27] J.M. Lee, Introduction to Smooth Manifolds, 2nd ed., Graduate Texts in Mathematics 218, Springer-Verlag New York, 2012. MR2954043

[28] Ming Liao, Lévy processes in Lie groups, Cambridge Tracts in Mathematics, Cambridge University Press, 2004. MR2060091 
Lévy processes on smooth manifolds with a connection

[29] S. I. Marcus, Modeling and approximation of stochastic differential equations driven by semimartingales, Stochastics 4 (1981), no. 3, 223-245. MR0605630

[30] A. Mohari, Ergodicity of Lévy flows, Stochastic Processes and their Applications 112 (2004), no. 2, 245-259. MR2073413

[31] B. O'Neill, Semi-Riemannian geometry: with applications to relativity, Pure and applied mathematics 103, Academic Press, 1983. MR0719023

[32] M. Pontier and A. Estrade, Relèvement horizontal d'une semimartingale càdlàg, Séminaire de probabilités de Strasbourg 26 (1992), 127-145 (fr). MR1231989

[33] P. Protter, Stochastic Integration and Differential Equation, second ed., Springer-Verlag, Berlin, Heidelberg, 1992. MR1037262

[34] D. Revuz and M. Yor, Continuous Martingales and Brownian Motion , 3rd ed., Springer, 1999. MR1725357

[35] S. J. Rogerson, Stochastic dynamical systems and processes with discontinuous sample paths, Ph.D. thesis, University of Warwick, 1981.

[36] G. Rudolph and M. Schmidt, Differential Geometry and Mathematical Physics: Part II. Fibre Bundles, Topology and Gauge Fields, 1st ed., Theoretical and Mathematical Physics, Springer, 2017. MR3616138

[37] K. Sato, Lévy Processes and Infinitely Divisible Distributions, Cambridge Studies in Advanced Mathematics, Cambridge University Press, 1999. MR1739520

[38] Alexander Schnurr, On the semimartingale nature of Feller processes with killing, Stochastic Processes and their Applications 122 (2012), no. 7, 2758-2780. MR2926174

Acknowledgments. We thank David Applebaum for helpful comments, which greatly improved an earlier version of the paper. This paper forms a part of the PhD thesis of the second author and we would like to thank the examiners Andreas Kyprianou and Kenneth David Elworthy for thorough reading and in-depth comments which greatly improved the contents of the paper. 


\section{Electronic Journal of Probability Electronic Communications in Probability}

\section{Advantages of publishing in EJP-ECP}

- Very high standards

- Free for authors, free for readers

- Quick publication (no backlog)

- Secure publication $\left(\mathrm{LOCKSS}^{1}\right)$

- Easy interface (EJMS²)

\section{Economical model of EJP-ECP}

- Non profit, sponsored by $\mathrm{IMS}^{3}, \mathrm{BS}^{4}$, ProjectEuclid ${ }^{5}$

- Purely electronic

\section{Help keep the journal free and vigorous}

- Donate to the IMS open access fund ${ }^{6}$ (click here to donate!)

- Submit your best articles to EJP-ECP

- Choose EJP-ECP over for-profit journals

\footnotetext{
${ }^{1}$ LOCKSS: Lots of Copies Keep Stuff Safe http://www. lockss.org/

${ }^{2}$ EJMS: Electronic Journal Management System http://www.vtex.lt/en/ejms.html

${ }^{3}$ IMS: Institute of Mathematical Statistics http://www.imstat.org/

${ }^{4}$ BS: Bernoulli Society http://www. bernoulli-society.org/

${ }^{5}$ Project Euclid: https://projecteuclid.org/

${ }^{6}$ IMS Open Access Fund: http://www.imstat.org/publications/open.htm
} 